\title{
Dynamic Decoupling and Intelligent Optimal PID Controller Tuning of Multivariable Qua-drones
}

\author{
Dong Hwa Kim
}

\begin{abstract}
This paper deals with dynamic decoupling and its intelligent PID control method of multivariable qua-drone. Up to this time, many sophisticated intelligent algorithms and control methods for drone systems have been mentioned. However, almost many cases have been focusing on single loop control methods and general multivariable systems. Therefore, we cannot guarantee its stability and optimal response by PID control used in multivariable qua-drone. Herein, this paper suggests a novel control method for PID control for multivariable qua-drone. As first step, this paper decouples dynamic of multivariable quadrone using diagonal method of system matrix and then applies intelligent method PSO and GA to single loop obtained by decoupling method to obtain optimal response.
\end{abstract}

Keyword: Drone Control, Genetic Algorithm, Pso, ZieglerNichols, Multivariable, Intelligent Control.

\section{I.INTRODUCTION}

$\mathrm{R}$ ecently, qua-drone (quadrotor) has been usefully using in many fields such as delivery, agriculture, education for high technology as well as military. High-tech in MEMO sensors, AI chips, and new control theory have motivated applications as well as research for qua-drone. Applying of drone has more potential such as transportation, entertainment, sports, safe and environment survey, and others as UAV (Unmanned Aerial Vehicle). To do that, there are many things that we must overcome the fundamental problem with safe operation, stabilization, robustness in the environment, and autonomous operation depends on trajectory tracking capabilities. Herein, it is important to study for a novel control strategies and analysis of dynamic behavior. As qua-drone (quarotor) is one of UAV (Unmanned Aerial Vehicle), which is operated by the full range of motion propelled by four (or six) rotors symmetrically. Because it's design and structure can be flexibly changed, safety and control mechanisms also must be analyzed to guarantee for operation with inexpensive and easy to design and assemble. As the qua-drone has one of the complex robot dynamics, we can usefully apply complex robot dynamics to research a fundamental or platform related to autonomous mobility and perception or aerial robotics [3-5].

Manuscript received on November 20, 2021.

Revised Manuscript received on November 25, 2021.

Manuscript published on November 30, 2021.

* Correspondence Author

Dong Hwa Kim, Prof., Department of Electrical Power and Control Engineering, Hanbat National University, South Korea. E-mail: koreahucare@gmail.com

(C) The Authors. Published by Blue Eyes Intelligence Engineering and Sciences Publication (BEIESP). This is an open access article under the CC BY-NC-ND license (http://creativecommons.org/licenses/by-nc-nd/4.0/)
Because of these capabilities and applications, currently, many have been researching quadrotor design and application as well as operation for a multidisciplinary engineering education in undergraduate course and technical high schools [17, 35, 44]. Basically, the qua-drone is a highly nonlinear and multivariable system that is strongly coupled and has an unstable system. To overcome this complexity of the control in different behavior conditions of qua-drone, many control approaches have been applying by title such as PID controllers [4, 21-22], fuzzy-PID control [18], Neural network-PID method [12], discrete-PID [28], sliding mode control [13], feedback linearization [25, 26], adaptive control [15], A Robust $\mathrm{H} \infty$ control [29] There are many types of qua-drone configurations according to the orientation of the blades and to the body coordinate system, such as tri-copter, quad-copter, v-tail, penta-copter, hexacopter. The quad-copter (qua-drone) translational motion depends on the tilting of the rotorcraft platform towards the desired orientation. Hence, engineers should consider that the translational and rotational motion are tightly coupled and the change of rotating speed of one rotor causes a motion in three degrees of freedom. As I mentioned before, as qua-drone is dynamically unstable as well as highly nonlinear and multivariable system, proper control is necessary to make it stable. Its instability comes from the changing rotorcraft parameters and environmental disturbances such as wind and speed [21, 22]. Besides, the cross-coupling between rotors (degrees of freedom) make it very sensitive to disturbances. On the other hand, the PID controller has an important role in industrial fields such as, chemical process, motor control, power plant, water purification, and so on because all systems in industry should have controller to obtain safety and optimal target. For this purpose, the proven PID controller has been used because it is not only due to the simple structure which is conceptually easy to understand but also to the tuning algorithm that provides adequate performance in the vast majority of applications using simple theory. For its optimum control, many approaches have been applied to provide simple tuning formula for determining the controller parameters. However, they do not provide good tuning or produce a satisfactory closed-loop response for many situations as well as complex processes or multivariable systems like drones because of computing speed. Because of these difficulties, the engineers need to have a highly tuning technique for a required performance and the controllers are rarely tuned optimally [6-8]. To improve the performance of the tuning function for systems with changing dynamic properties of drones, several intelligent tuning strategies have been proposed, for example, fuzzy based tuning [11] and neural network-based tuning [8].

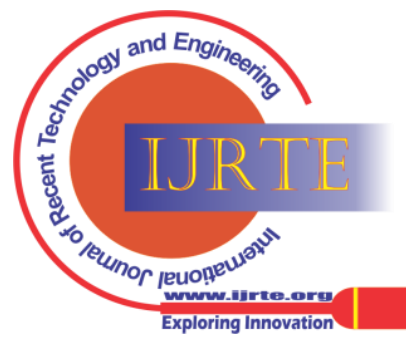


That is recently, there has been growing interest in using intelligent approaches such as fuzzy, neural network, evolutionary method, fuzzy neural network [13], clonal selection algorithm [31], and bacterial foraging approach [30]. Herein, to provide consistent, reliable, safe and optimal solution to industrial control problems, and advanced intelligent tuning schemes are needed. Currently, PID controllers have been trying to be applied in quadrotor and UAV as a single loop not multivariable. This paper provides the intelligent tuning PID controller for qua-drone with a highly nonlinear system and strongly coupled multivariable characteristics. Firstly, this paper develops and computes coupling index (interacting index) through dynamic equations of qua-drone. Secondly, by using this index, we adjust the interacting level by GA and PSO. Depending on the level of interacting index, we also tune the PID controller's parameters by GA and PSO to obtain optimal response that we want. Usually, in case of multivariables, because of interacting between parameters, tuning is not easy as engineers. This paper offers an adjusting method of parameters with intelligent tuning.

\section{II.LITERATURE REVIEW OF MULTIVARIABLE DRONE CONTROLS}

Ref. [66] provide LQ and LQG methodologies for quadcopter control system. The developed control system is for both the rectangular position $(\mathrm{x}, \mathrm{y})$ and altitude $(\mathrm{z})$ as well as the orientation based on 6-Degree of Freedom (6DOF) mathematical model. This paper designs altitude and attitude controllers using a nonlinear mathematical model obtained first from 6DOF. The control goal has also function to track a spatial trajectory with the quadcopter center of gravity under environment disturbances and sensor measurement errors. Ref. [64] presents an analysis and performance of a LQR control algorithm for quadrotor helicopters. First, they have dealt with the dynamic model for the quadcopter for a successful analysis and then the controller designed, tuned. For tuning, they introduce the LQR verified in terms of delay time, rise time, overshoot, settling time and tolerance limits. This paper [65] designs an infinite-horizon adaptive linear quadratic tracking (ALQT) control scheme for optimal attitude tracking of a quadrotor unmanned aerial vehicle (UAV) to obtain experimentally validated in the presence of real-world uncertainties in quadrotor system parameters and sensor measurement. However, they do not mention about multivariable system of the designed control scheme. This paper [67] illustrates an adaptive control technique to stabilize the attitude dynamics of UAV (unmanned aerial vehicle) type quadrotor in the presence of disturbances and/or uncertainties in the parameters due to changes in the payload, nonlinear actuators, and change in environmental conditions. To obtain address the above problem, this paper uses MRAC (model reference adaptive control) strategy using a cost function that defined as a function of the error between the output of the system and a desired response from a reference model. This paper uses Lyapunov's theory to guarantee the global asymptotic stability of the system and simulates using MATLAB-Simulink. This paper [68] shows two controllers that simultaneously coordinates the position and orientation motions of a quadrotor to enable the minimization of

the overshooting. To do that, this paper has a two controller. The first controller is based on Euler angles and the second on quaternions.

The paper [23] focuses on mathematical modelling of a quadrotor and identification of parameters of the quadrotor to design a controller. They define drone dynamic in terms of quaternions through the body-fixed frame, the inertial frame, mathematical model near a hover position.

As multivariable system, Ref [69] deals with multivariable (MIMO) control loops by simulation for the two-variable controlled plant of a quadruple-tank process not in drone. They decide determine the optimal control pairs using the relative gain array as the condition number or the Niederlinski index. This article [70] offers an information fusion based decoupling control (IFBDC) system for the nonlinear coupled multiple-input multipleoutput systems. Test is done on a robot manipulator system to investigate the effectiveness of the decoupling control strategy and to analyze its key parameters and the computational cost. The main objective of this work [25] is to implement a control law capable of decoupling the system for a small-scale unmanned helicopter. This paper provides the active disturbance rejection control by combining decoupling and disturbance rejection without a detailed plant dynamic. This paper [63] has focuses on developing a comprehensive theory for the decoupling of multivariable systems by state feedback. This paper begins by giving a preliminary formulation of the decoupling problem, discussing certain aspects of its solution, reviewing previous research, and indicating the contributions of this paper.

This author [69] focuses a simple analytical design method of decoupling controller matrix is proposed in terms of idea of coupling matrix for two-input-two-output (TITO) processes with time delays in chemical and industrial practice based on unit feedback closed-loop control structure. And they design PID decoupling controller analytically by means of powerful robustness of two degree-of-freedom PID Desired Dynamic Equation (DDE) method, and introduce the Monte-Carlo stochastic experiment to analyze performance robustness of the controller. In multivariable system design, it is a quite important to understand that the Nyquist criterion is a valuable design tool with applications to control systems and circuits [24, 27]. We can decide decoupling level of multivariable by the precise shapes of several Nyquist curves and relate them to the shapes of the classical plane curves. There are many research approaches in drone controller design and tuning Ref. [33] gives information about a tutorial of the platform configuration, methodology of modeling, comprehensive nonlinear model, the aerodynamic effects, and model identification for a quadrotor, as review paper. Ref. [38] provides information of PID controller design for quadrotor that has a reliable nonlinear dynamic model of the quadrotor. They have the simplified nonlinear dynamic model and proposed the PID controllers with feedback linearization and feedforward control using the backstepping method. They use these controllers to control both the attitude and position of the quadrotor.

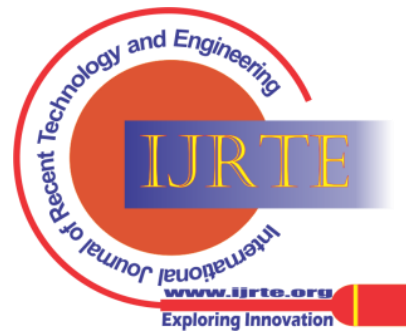


Ref. [17] is to apply LQ and LQG methodologies for quadcopter control system. For that, they developed 6-DOF mathematical model with both the rectangular position $(\mathrm{x}, \mathrm{y})$ and altitude (z) as well as the orientation (attitude - angles around the axes). Ref. [18] describes kinematics by an effective use of Jacobi method to derive equations with respect to the body frame of reference and inertial frame of reference. Solutions have been obtained for important parameters such as linear acceleration, angular acceleration and torque.

Ref [70] paper presents an altitude control algorithm for quadcopters that consists of a combination of nonlinear and linear controllers. They prescribe certain flight conditions through the Lyapunov theory and compare the control performance of their control method to that of a conventional PID on simulation.

Ref. [11, 20] proposes the use of interval type-2 fuzzy for controlling the altitude of a nonlinear quadcopter UAV using Gaussian membership function and utilizing the Enhance Iterative Algorithm with Stop Condition (EIASC) algorithm for type-reduction. A comparison between the proposed interval type-2 fuzzy controller and proportionalDerivative (PD) controller is illustrated. Simulation results demonstrated that the tracking performance of the proposed controller outperformed the PD controller.

Ref. [68] proposed a neural network approach for modelling small unmanned vehicle's trajectory without knowing its control system and bypassing exhaustive efforts for aerodynamic parameter identification. This work used the trajectory data generated by a mathematical vehicle model for training and testing the neural network.

Ref. [38] propose PID and Fuzzy Logic for UAV. That is considered as both Offline and Online Trained Neural Network controllers that is crucial to achieve hover stability.

Ref. [39, 41, 42] aimed to obtain an artificial intelligencebased controller for quadrotors. Firstly, the quadrotor is modeled according to quadrotor dynamics. Then, PD controllers for $\mathrm{x}, \mathrm{y}$, yaw and $\mathrm{z}$ control of quadrotor are implemented as classical controllers.

The results for these controllers are recorded as training data of NN controllers. NN controllers are trained according to these data and achieve good trajectory tracking results. Ref. [43] present a method to control a quadrotor with a neural network trained using reinforcement learning techniques. With reinforcement learning, a common network can be trained to directly map state to actuator command making any predefined control structure obsolete for training.

\section{III.DYNAMIC DECOUPLING AND EVOLUTIONARY- BASED INTELLIGENT OPTIMAL PID TUNING OF MUTIVARIABLE QUADRONES}

\section{A. Multivariable Qua-drone Dynamics for Controller Design}

The quad-drone is a UAV built from four rotors installed at the drone structure (tri-copter, quad-copter, v-tail, pentacopter, hexa-copter). Each rotor consists of a fixed-pitch propeller mounted to the motor for lifting the vehicle while controlling its attitude simultaneously.

Quad-drone is also popular as a research platform in flight control areas due to the simple design and maintenance; the ability and stability to take- off, hover, and land vertically; being inexpensive and of lower risk compared to traditional helicopter [3, 32].

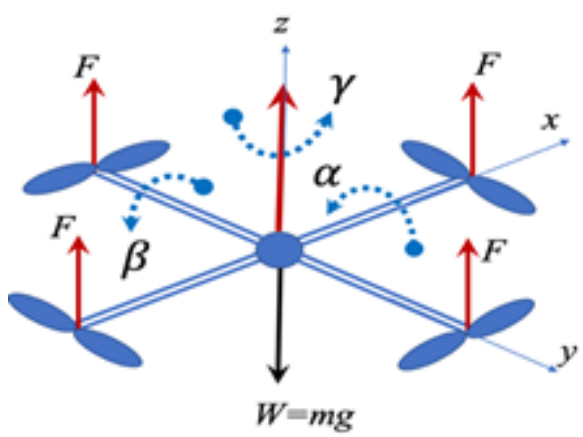

Figure. 1. Qua-drone structure

The structure of the qua-drone is illustrated as shown in Fig. 1. The latitude $(z)$ of hovering is decided through rolling $(\alpha)$, pitching $(\beta)$, and yawing $(\gamma)$ and speed is directly actuated by changing the rotor's RPM.

Conventionally, the dynamic model of a qua-drone was derived from the Newton-Euler equation of motion; the rotational dynamics of transformation of a robot can be expressed by the following moving vector equation [34]:

$$
\Gamma \mathcal{W}=u-\omega \times(\Gamma \omega)
$$

In equation (1), $\mathcal{W}$ : the qua-drone's angular acceleration vector, $u$ : the control torque vector, $\omega$ : the current angular rate, the inertia tensor: $\Gamma$. The inertia tensor, $\Gamma$ consists of three inertia terms with respect to $x, y$, and $z$ rotation axis. That is, the position vector is defined by $A_{x x}, A_{y y}$, and $A_{z z}$.

$$
\Gamma=\left[\begin{array}{ccc}
A_{x x} & A_{x y} & A_{x z} \\
A_{y x} & A_{y y} & A_{y z} \\
A_{z x} & A_{z y} & A_{z z}
\end{array}\right]
$$

When the qua-drone angular rate $\omega$ consists of the rolling rate $\mathrm{R}$, pitching rate $\mathrm{P}$, and yawing rate $\mathrm{Y}$. the quadrone's current angular rate is defined by $\omega=\left[\begin{array}{lll}R & P & Y\end{array}\right]^{T}$. The qua-drone control torque vector, $\mathrm{u}$, consists or rolling torque $u_{R}$, pitching torque, $u_{P}$, yawing torque $u_{Y}$. The control vector is given by $u=\left[\begin{array}{lll}u_{R} & u_{P} & u_{Y}\end{array}\right]^{T}$.

Newton-Euler formalism for the quadrotor attitude dynamics equation as follows:

$$
\begin{aligned}
& \frac{d R}{d t}=\frac{A_{y y}-A_{z z}}{A_{x x}} P Y-\frac{\Gamma_{\text {rotor }}}{A_{x x}}+\frac{u_{R}}{A_{x x}} \\
& \frac{d P}{d t}=\frac{A_{z z}-A_{x x}}{A_{y y}} R Y-\frac{\Gamma_{\text {rotor }}}{A_{y y}} \\
& \frac{d Y}{d t}=\frac{A_{x x}-A_{y y}}{A_{z z}}+\frac{u_{Y}}{A_{z z}} \\
& \frac{d}{d t} \frac{d z}{d t}=-g+\{\cos (\alpha) \cos (\beta)\} \frac{u_{z}}{m}
\end{aligned}
$$

Published By:

Blue Eyes Intelligence Engineering and Sciences Publication (BEIESP) 


$$
\dot{X}=F(X, U)=\left[\begin{array}{c}
\phi_{2} \\
\theta_{2} \psi_{2} a_{1}+\theta_{2} \Omega_{r} a_{2}+b_{1} U_{\phi} \\
\theta_{2} \\
\phi_{2} \psi_{2} a_{3}-\phi_{2} \Omega_{r} a_{4}+b_{2} U_{\theta} \\
\psi_{2} \\
\phi_{2} \theta_{2} a_{5}+b_{3} U_{\psi} \\
z_{1} \\
g-\frac{U_{1}}{m}\left(\cos x_{1} \cos x_{3}\right) \\
x_{1} \\
\frac{U_{1}}{m}\left(\sin x_{1} \sin x_{5}+\cos x_{1} \sin x_{3} \cos x_{5}\right) \\
y_{2} \\
\frac{U_{1}}{m}\left(\sin x_{1} \cos x_{5}+\cos x_{1} \sin x_{3} \sin x_{5}\right)
\end{array}\right]
$$

where $X=\left[\phi_{1}, \phi_{2}, \theta_{1}, \theta_{2}, \psi_{1}, \psi_{2}, z_{1}, z_{2}, x_{1}, x_{2}, y_{1}, y_{2}\right]$ is a state vector with roll, pitch, yaw, and height variable and $a_{1}, a_{2}, a_{3}, a_{4}, a_{5}, b_{1}, b_{2}, b_{3}$ are constants.

The orientation of the qua-drone can be measured as the rolling, pitching, and yawing when qua-drone flights with variation of angles $(\alpha, \beta, \gamma)$ and its position is given by their differential with respect to time related to $\mathrm{R}, \mathrm{P}, \mathrm{Y}$, as equation(4) [37].

Consequently, a final model of altitude dynamics can be defined by roll, pitch, and yaw and its coupling functions, each other. Thus, controlling altitude dynamics is strongly connected with the control of rolling, pitching, and yawing. Herein, because these conditions are nonlinear and multivariable systems, it is difficult for multivariable systems to control by PID control.

B. Dynamic State Equation and Decoupling of Multivariable Qua-drone Model

When using equation (4) for qua-drone, the state model of qua-drone is $12 \times 12$ model [3]:

$$
\begin{aligned}
& X^{*}=[A] X+[B] U \\
& \mathrm{Y}=[\mathrm{C}] \mathrm{X}+[\mathrm{D}] \mathrm{U}
\end{aligned}
$$

Hence, system matrix is

$$
\mathbf{A}=\left[\begin{array}{cccccccccccc}
0 & 0 & 0 & 0 & 0 & 0 & 0 & 0 & 0 & 0 & -g_{0} & 0 \\
0 & 0 & 0 & 0 & 0 & 0 & 0 & 0 & 0 & g 0 & 0 & 0 \\
0 & 0 & 0 & 0 & 0 & 0 & 0 & 0 & 0 & 0 & 0 & 0 \\
0 & 0 & 0 & 0 & 0 & 0 & 0 & 0 & 0 & 0 & 0 & 0 \\
0 & 0 & 0 & 0 & 0 & 0 & 0 & 0 & 0 & 0 & 0 & 0 \\
0 & 0 & 0 & 0 & 0 & 0 & 0 & 0 & 0 & 0 & 0 & 0 \\
1 & 0 & 0 & 0 & 0 & 0 & 0 & 0 & 0 & 0 & 0 & 0 \\
0 & 1 & 0 & 0 & 0 & 0 & 0 & 0 & 0 & 0 & 0 & 0 \\
0 & 0 & 1 & 0 & 0 & 0 & 0 & 0 & 0 & 0 & 0 & 0 \\
0 & 0 & 0 & 1 & 0 & 0 & 0 & 0 & 0 & 0 & 0 & 0 \\
0 & 0 & 0 & 0 & 1 & 0 & 0 & 0 & 0 & 0 & 0 & 0 \\
0 & 0 & 0 & 0 & 0 & 1 & 0 & 0 & 0 & 0 & 0 & 0
\end{array}\right]
$$

$\mathbf{B}=\left[\begin{array}{cccc}0 & 0 & 0 & 0 \\ 0 & 0 & 0 & 0 \\ -0.0307 & -0.0310 & -0.0421 & -0.0307 \\ 0 & 1.0851 & 0 & -1.0743 \\ 1.0742 & 0 & -1.4713 & 0 \\ 0.0483 & -0.0488 & 0.0661 & -0.0483 \\ 0 & 0 & 0 & 0 \\ 0 & 0 & 0 & 0 \\ 0 & 0 & 0 & 0 \\ 0 & 0 & 0 & 0 \\ 0 & 0 & 0 & 0 \\ 0 & 0 & 0 & 0\end{array}\right]$

$$
\mathbf{C}=\left[\begin{array}{cccccccccccc}
0 & 0 & 0 & 0 & 0 & 0 & 0 & 0 & 0 & 0 & 0 & 0 \\
0 & 0 & 0 & 0 & 0 & 0 & 0 & 0 & 0 & 0 & 0 & 0 \\
0 & 0 & 0 & 0 & 0 & 0 & 0 & 0 & 0 & 0 & 0 & 0 \\
0 & 0 & 0 & 1 & 0 & 0 & 0 & 0 & 0 & 0 & 0 & 0 \\
0 & 0 & 0 & 0 & 1 & 0 & 0 & 0 & 0 & 0 & 0 & 0 \\
0 & 0 & 0 & 0 & 0 & 1 & 0 & 0 & 0 & 0 & 0 & 0 \\
0 & 0 & 0 & 0 & 0 & 0 & 0 & 0 & 0 & 0 & 0 & 0 \\
0 & 0 & 0 & 0 & 0 & 0 & 0 & 0 & 0 & 0 & 0 & -1 \\
0 & 0 & 0 & 0 & 0 & 0 & 0 & 0 & 0 & 0 & 0 & 0
\end{array}\right]
$$$$
\mathbf{D}=\left[\begin{array}{cccc}
-0.0559 & 0 & 0.0765 & 0 \\
0 & 0.0564 & 0 & -0.0559 \\
0 & 0 & 0 & 0 \\
0 & 0 & 0 & 0 \\
0 & 0 & 0 & 0 \\
0 & 0 & 0 & 0 \\
0 & 0 & 0 & 0 \\
0 & 0 & 0 & 0 \\
0 & 0 & 0 & 0
\end{array}\right]
$$

\section{Decoupling Condition of multivariable Qua-drone}

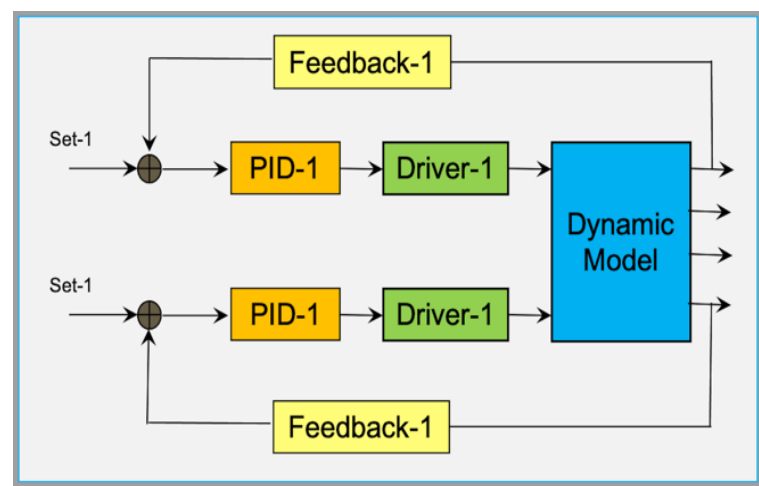

Figure. 2. Block-diagram of decoupled control system 


$$
D_{I 2}(s)=-\frac{G_{12}(s)}{G_{I I}(s)} \quad \text { and } \quad D_{21}(s)=-\frac{G_{21}(s)}{G_{22}(s)}
$$

There are many decoupler design method [21-24] but this paper uses a feed-forward decoupling controller condition [20] to make a simple design as shown in Figure 3 and equation (9).

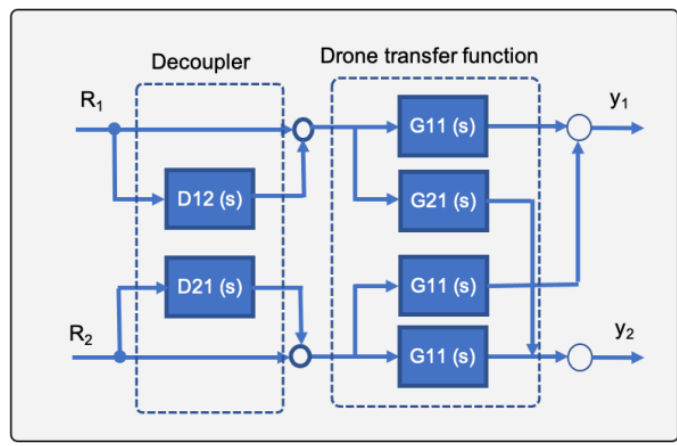

Figure 3. Decoupler condition of Fig. 10

In $2 \times 2$ multivariable process, the following condition $\left(\mathrm{S}_{0}, \ldots, \mathrm{S}_{\mathrm{n}}\right)$ is obtained by integrating the process of the open loop step response $\mathrm{y}(\mathrm{t})$ after applying the step-change $d u$ of the process input at $=0$ [33]:

$$
\begin{gathered}
S_{0}=y_{0}(\infty) \\
\vdots \\
S_{n}=y_{n}(\infty)
\end{gathered},
$$

Where,

$$
\begin{gathered}
y_{0}(t)=\frac{y(t)-y(0)}{\Delta u} \\
\vdots \\
y_{n}(t)=\int_{0}^{t}\left[S_{n-1}-y_{n-1}(\tau)\right] d \tau
\end{gathered}
$$

and $S_{0}$ is the steady-state gain of the process. If the process is known and given by the following transfer function:

$$
G(s)=k_{c} \frac{1+b_{1} s+b_{2} s^{2}+\ldots+b_{m} s^{m}}{1+a_{1} s+a_{2} s^{2}+\ldots+a_{n} s^{n}} e^{-s L},
$$

then, it can be characterized by the following areas [34]:

$$
\begin{aligned}
S_{0}= & k_{c} \\
S_{1}= & k_{c}\left(a_{1}-b_{1}+L\right) \\
S_{2}= & k_{c}\left(b_{2}-a_{2}-L b_{1}+\frac{L^{2}}{2 !}\right)+S_{1} a_{1} \\
S_{n}= & k_{c}\left((-1)^{n+1}\left(a_{k}-b_{k}+\sum_{i=1}^{n}(-1)^{n+i} \frac{L^{i} b_{k-i}}{i !}\right) .\right. \\
& +\sum_{i=1}^{n-1}(-1)^{n+i-1} S_{i} a_{k-i}
\end{aligned}
$$

Equation given by Eq. (9) can be computed in the timedomain from the process steady-state change [34]. This paper tunes the gain of PID controller in each loop as well as calculates coupling gain $S_{n}$ depending on dynamic change online using evolutionary based tuning.

Thus, regardless of the numerous methods available, PID tuning was not really a simple task. Similarly, in the practical world, qua-drone practitioners found that the tuning of qua-drone's PID was difficult [27]. Hence, they tuned their PID by using a trial and error method [27-30]. It means it is not easy to tune by PID controller for quadrone.

To decouple multivariable qua-drone, this paper multiplies arbitrary matrix $12 \times 12$ to system matrix [A] of equation (5a). This paper performs several time multiplications to the system matrix [A]. Then we can obtain a diagonal matrix. It means the system is decoupled.

\section{PID Control Law of Multivariable Qua-drones}

To control the Qua-drone under decoupled multivariable of qua-drone, a PID control technique has been used because of its easy implementation and simple theory for design. Because the feedback signal of the PID controller produces the error of the output and desired error, we define an error as follows:

$\mathrm{e}=\mathrm{x}_{\mathrm{d}}-\mathrm{x}$

Where, $e$ is the error between the desired reference and output. This error is defined for all controllable angles and height. The control law of the PID controller is to obtain gains, $K_{\mathrm{p}}, K_{\mathrm{i}}$, and $K_{\mathrm{d}}$ as illustrated in Fig. 4 and Equation (6):

$u(t)=K e(t)+K_{i} \int_{0}^{t} e(t) d t+K_{d} \frac{d e(t)}{d t}$

For the height control, the error should be defined as $e_{h}=z_{1}-z_{d}$ because the control input appears negatively in the model. Here, the PID controller controls each input of qua-drone, $\alpha, \beta, \gamma$, and $\mathrm{z}$ as shown in Figure 5. There are strong inter-actions among the parameters of qua-drone such as the latitude $(z)$, rolling $(\alpha)$, pitching $(\beta)$, and yawing $(\gamma)$ illustrated in Fig. 3. Therefore, it is very difficult to control by the traditional PID controller because of interaction of multivariable systems as shown in Figure 6.

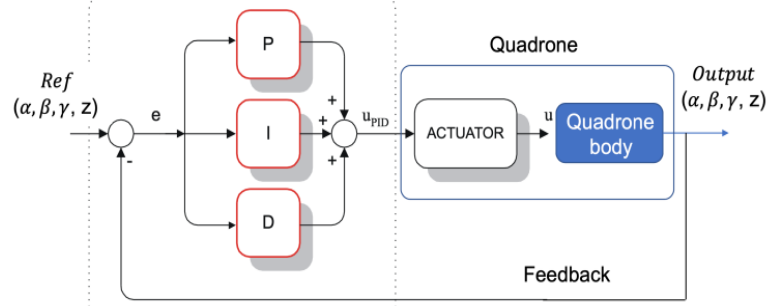

Figure 4. PID control structure of Qua-drone.

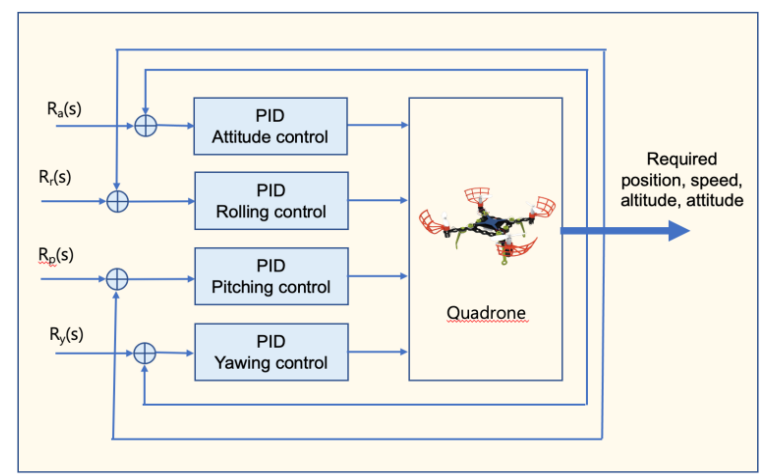

Figure. 5. PID control structure of Qua-drone.

\section{Published By:}

Blue Eyes Intelligence Engineering and Sciences Publication (BEIESP) 


\section{Dynamic Decoupling and Intelligent Optimal PID Controller Tuning of Multivariable Qua-drones}

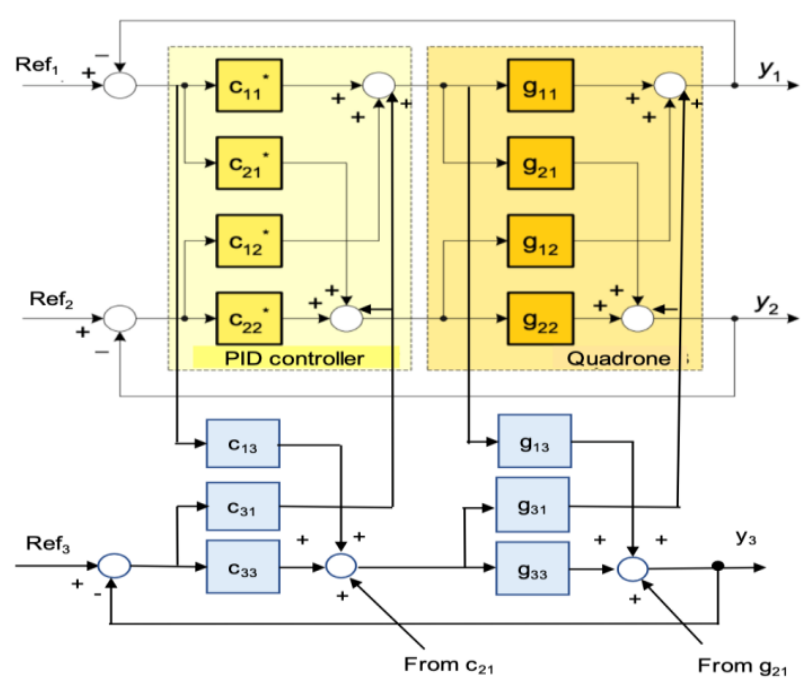

Figure. 6. Multivariable PID control structure of quadrone with $3 \times 3\left(\right.$ ref $_{1}$, ref $_{2}$, and ref $\left._{3}\right)$.

\section{Overall Control Law of Multivariable Qua-drones for Intelligent Optimal PID controller Tuning}

The PID controller of qua-drone is performed by the error values to generate the control torsion $[1,3,4,7-10]$ as shown in Figure 5 and 6. This is why they should use Newton-Euler model to analyze the dynamic behavior of the quadrotor flight.

When we refer to the symbols $\alpha, \beta$, and $\gamma$ for angles of roll, pitch, and yaw of the flight, respectively, $u \alpha, u \beta$, and $\mathrm{u} \gamma$ denote the torsion of the rolling, pitching, and yawing for the hovering of flight.

By using this definition, the PID controller scheme given in Figure 5 and 6 has as the following [21-25]:

$$
\begin{aligned}
& u_{z}=K_{p z}\left(z_{r}-z\right)+K_{i z} \frac{1}{s}\left(z_{r}-z\right)+K_{d z} \frac{d}{d t}\left(z_{r}-z\right) \\
& u_{\alpha}=K_{p \alpha}\left(\alpha_{r}-\alpha\right)+K_{i \alpha} \frac{1}{s}\left(\alpha_{r}-\alpha\right)+K_{d \alpha} \frac{d}{d t}\left(\alpha_{r}-\alpha\right) \\
& u_{\beta}=K_{p \beta}\left(\beta_{r}-\beta\right)+K_{i \beta} \frac{1}{s}\left(\beta_{r}-\beta\right)+K_{d \beta} \frac{d}{d t}\left(\beta_{r}-\beta\right) \\
& u_{\gamma}=K_{p \gamma}\left(\gamma_{r}-\gamma\right)+K_{i \gamma} \frac{1}{s}\left(\gamma_{r}-\gamma\right)+K_{d \gamma} \frac{d}{d t}\left(\gamma_{r}-\gamma\right)
\end{aligned}
$$

From the above equation, the PID controller compares the errors of the roll, pitch, yaw, and altitude with the given references. The commands of the controller control the hovering thrust; the rolling; pitching; and yawing control torsion for required position and speed.

From their trial and error approach, these qua-drone practitioners developed systematic steps that differ from one to another. Beginning from the rough method, the qua-drone flight dynamics were observed, and then the PID gains are adjusted until the oscillation disappears [30-36] and continued with the common procedure which tuned the pitch and roll parameters with the same values and then tuned the yaw [38]. Another separated technique is tuning one axis at a time, from the roll, pitch, and then yaw axis, while the quadrotor hovers. On that axis, they adjust each gain of $K_{\mathrm{P}}$, $K_{\mathrm{i}}$, and $K_{\mathrm{d}}$. Due to the coupling dynamics of quadrotor, they return to readjust the gains of the previous axis [45]. The different sequence is also used by tuning of $K_{\mathrm{p}}$ first, then $K_{\mathrm{d}}$, and finally the $K_{\mathrm{i}}$ gains [50-52]. Initially, the gains were set to zero and then slowly increased until the qua-drone's oscillation was critically damped; after that, the tuning process was repeated until the quadrotor flies in a stable manner. A practitioner's method in tuning qua-drone's PID gains using computational software was reported in [53-55]. Although the computational software seemed to result in optimized gains, the first flight crashed due to the incorrect sampling rate, and extensive tuning had to be performed again to fly the quadrotor at the following attempt. Furthermore, the increment of the controller performance conducted by tuning the PID gains manually. These conditions have enhanced the requirement for an accurate model of the quadrotor's dynamic to correctly tune its PID gains. The manual tuning of PID still becomes a common preferable method in quadrotor control studies [1, 2,240, 41, 46]. Thus, in this work, the PID gains were manually tuned to work on the discrete form of controller [27, 60]:

The decoupler condition of system Figure 6 is given by equation 6 and equations 7 and 8 . As far as minimize $D_{21}$ and $\mathrm{D}_{12}$, each loop of MIMO can be controlled by independently PID controller without interaction in MIMO loop.

\section{E. Intelligent PID Tuning of the Multivariable Qua- drones by Evolutionary Method}

Tuning of the conventional PID controllers is very difficult when the system has strong interactions like quadrone with multivariable systems [26, 27, 33, 35, 59, 63, 66, 69]. A lot of tuning methods have been developed for multiinput multi-output (MIMO) PI controllers [66], [69]. The MIMO applications require an exact system model for the decoupling.

\section{IV.SIMULATION AND RESULTS}

Figure 7 is a block diagram for simulation of the decoupled multivariable qua-drone system used in this paper. The decoupled qua-drone multivariable system can be considered as single loop after decoupled process and we can tune as the PID controller of single loop.

This paper applies GA and PSO to this multivariable loop and compares these GA and PSO with Ziegler-Nichols method to confirm the results of tuning performance.

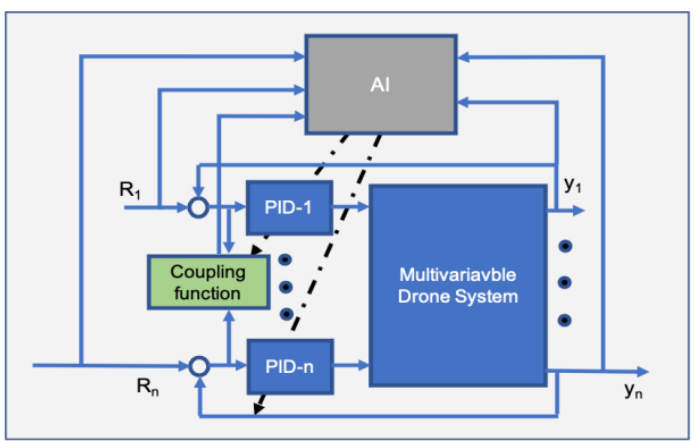

Figure. 7. The structure of AI based-PID control tuning of multivariable qua-drone

Published By:

Blue Eyes Intelligence Engineering and Sciences Publication (BEIESP)

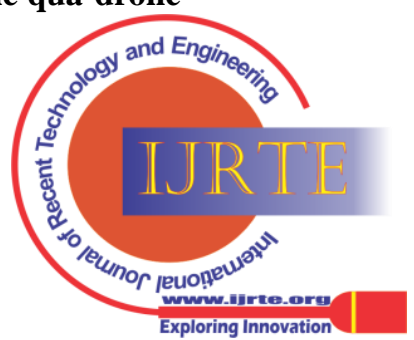




\section{A. Decoupling of Multivariable Qua-drone System}

Figure 8 shows system matrix A, B, C, and D obtained by the decoupling process.

By using these matrices, this paper obtains each single loop transfer function. That is, the transfer function of the decoupled multivariable qua-drone system is given by:

(10) From input 1 to output (System 1:0, System 2: 0, System 3: 0, System 4: $\left.1.976 / \mathrm{s}^{2}\right)$

(1) From input 2 to output (System $1: 2.895 / \mathrm{s}^{2}$ ), system 2: 0 , system 3: 0 , system 4: 0)

(1) From input 3 to output (System 1:0, system 2: 0, system 3: $\left.2.895 / \mathrm{s}^{2}\right)$, system $\left.4: 0\right)$

(1) From input 1 to output (System 1:0, system 2: 0, system 3: 0 , system $\left.4: 1.976 / \mathrm{s}^{2}\right)$.

\begin{tabular}{|c|c|c|c|c|c|c|c|}
\hline & $\mathrm{x} 1$ & $\mathrm{x} 2$ & $\times 3$ & $\times 4$ & x 5 & $x 6$ & $\times 7$ \\
\hline$x 1$ & 0 & 1 & 0 & 0 & 0 & 0 & 0 \\
\hline$x 2$ & 0 & 0 & 0 & 0 & 0 & 0 & 0 \\
\hline x3 & 0 & 0 & 0 & 1 & 0 & 0 & 0 \\
\hline$\times 4$ & 0 & 0 & 0 & 0 & 0 & 0 & 0 \\
\hline x5 & 0 & 0 & 0 & 0 & 0 & 1 & 0 \\
\hline$x 6$ & 0 & 0 & 0 & 0 & 0 & 0 & 0 \\
\hline$\times 7$ & 0 & 0 & 0 & 0 & 0 & 0 & 0 \\
\hline x8 & 0 & 0 & 0 & 0 & 0 & 0 & 0 \\
\hline
\end{tabular}

$\mathrm{B}=$

$\begin{array}{rrrrr} & u 1 & u 2 & u 3 & u 4 \\ \times 1 & 0 & 0 & 0 & 0 \\ x 2 & 0 & 2895 & 0 & 0 \\ \text { x3 } & 0 & 0 & 0 & 0 \\ \text { x4 } & 0 & 0 & 289.5 & 0 \\ \text { x5 } & 0 & 0 & 0 & 0 \\ \text { x6 } & 0 & 0 & 0 & 3838 \\ \text { x7 } & 0 & 0 & 0 & 0 \\ \text { x8 } & 1.976 & 0 & 0 & 0\end{array}$

$\mathrm{C}=$

$$
\begin{array}{rrrrrrrrr} 
& x 1 & x 2 & x 3 & x 4 & x 5 & x 6 & x 7 & x 8 \\
\text { y1 } & 1 & 0 & 0 & 0 & 0 & 0 & 0 & 0 \\
\text { y2 } & 0 & 0 & 1 & 0 & 0 & 0 & 0 & 0 \\
\text { y3 } & 0 & 0 & 0 & 0 & 1 & 0 & 0 & 0 \\
\text { y4 } & 0 & 0 & 0 & 0 & 0 & 0 & 1 & 0
\end{array}
$$$$
\mathrm{D}=
$$

$\begin{array}{rrrrr} & u 1 & 12 & u 3 & u 4 \\ \mathrm{~V} 1 & 0 & 0 & 0 & 0 \\ \mathrm{~V}^{2} & 0 & 0 & 0 & 0 \\ \mathrm{~V} 3 & 0 & 0 & 0 & 0 \\ \mathrm{~V} 4 & 0 & 0 & 0 & 0\end{array}$

Figure. 8. Matrix of decoupled multivariable qua-drone
Because these single loops have independent characteristics, the tuning of PID controller is easy and we can apply several tuning methods.

\section{B. Response of the Coupled Multivariable Qua-drone} system

Figure 8-17 shows the result of simulation in coupled system of qua-drone used in this paper.

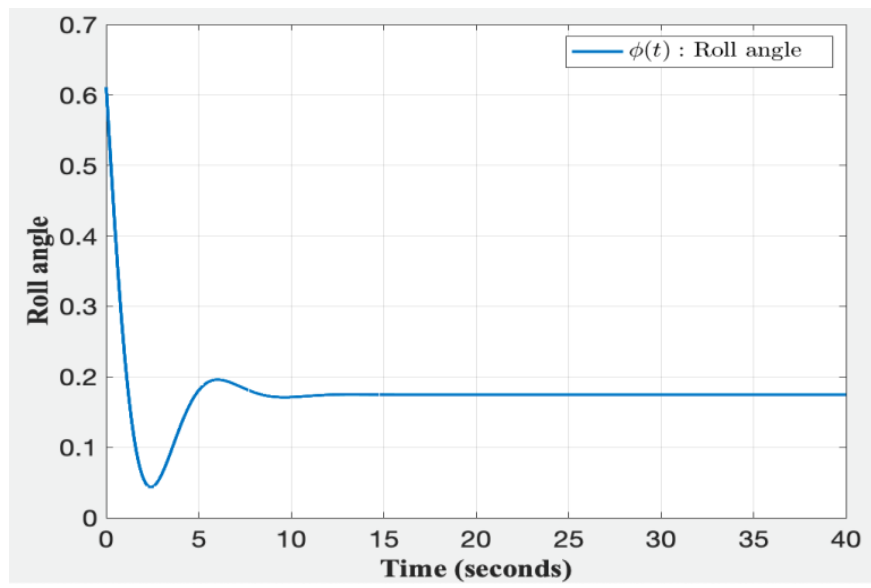

Figure. 9. Response of roll angle.

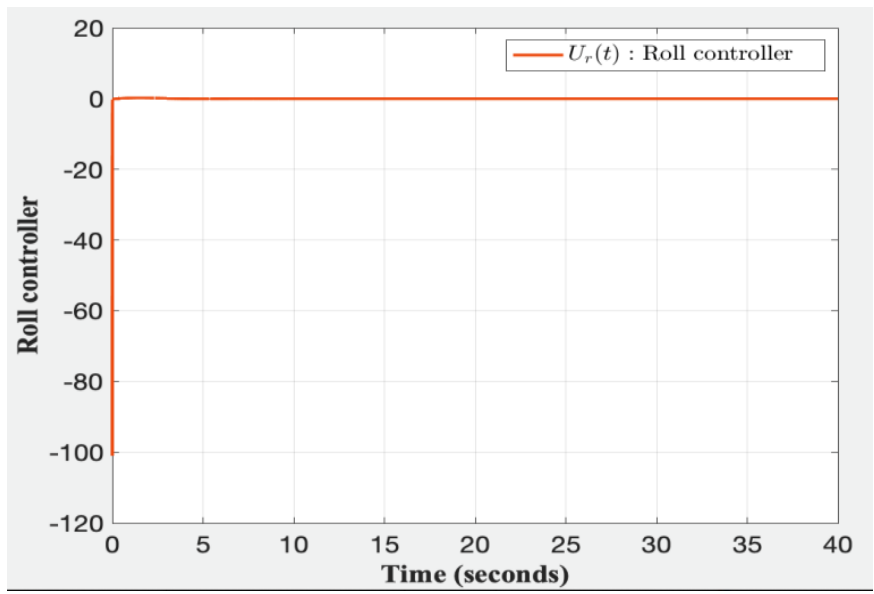

Figure. 10. Response of Roll controller.

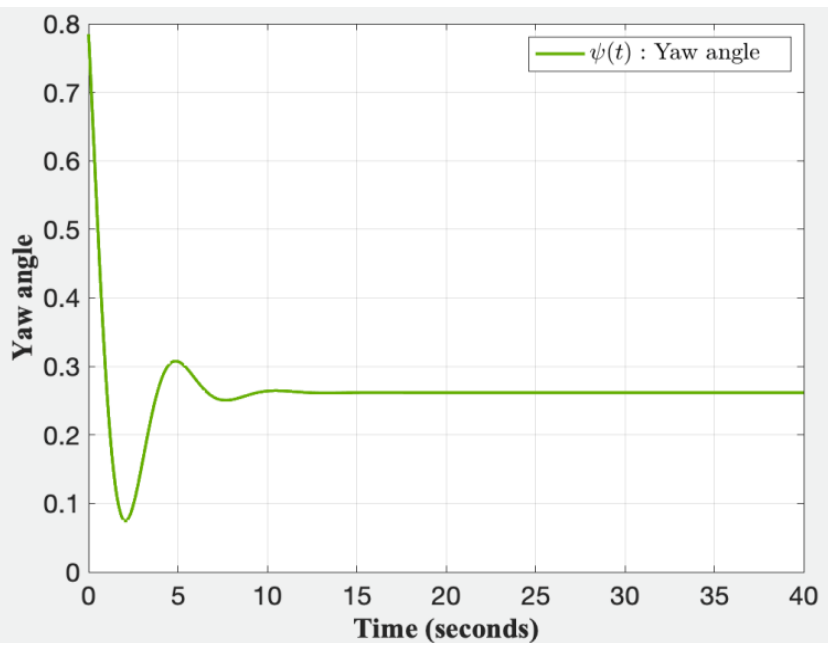

Figure. 11. Response of Yaw angle

Published By:

Blue Eyes Intelligence Engineering and Sciences Publication (BEIESP) 
Dynamic Decoupling and Intelligent Optimal PID Controller Tuning of Multivariable Qua-drones

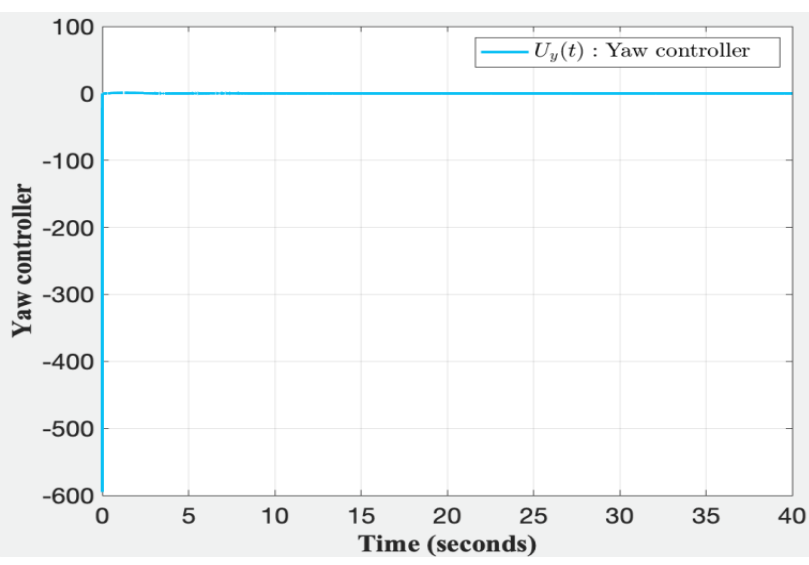

Figure. 12.Yaw controller

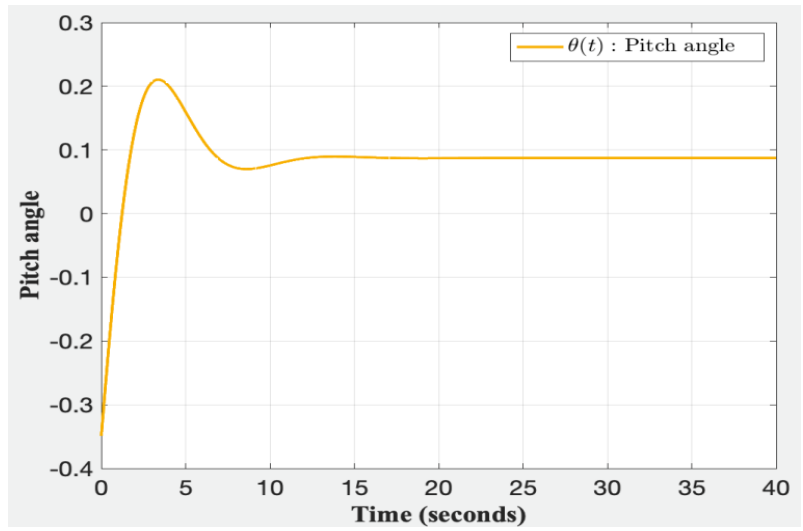

Figure. 13. Response of Pitch angle.

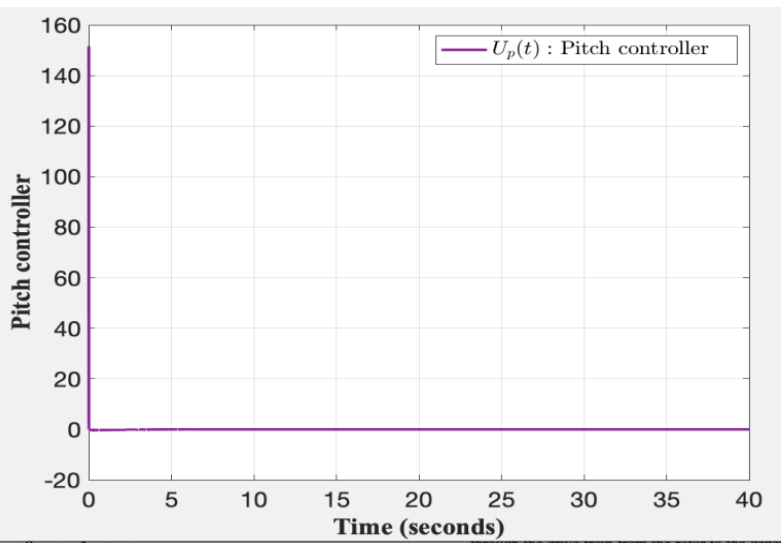

Figure. 14. Response of Pitch controller

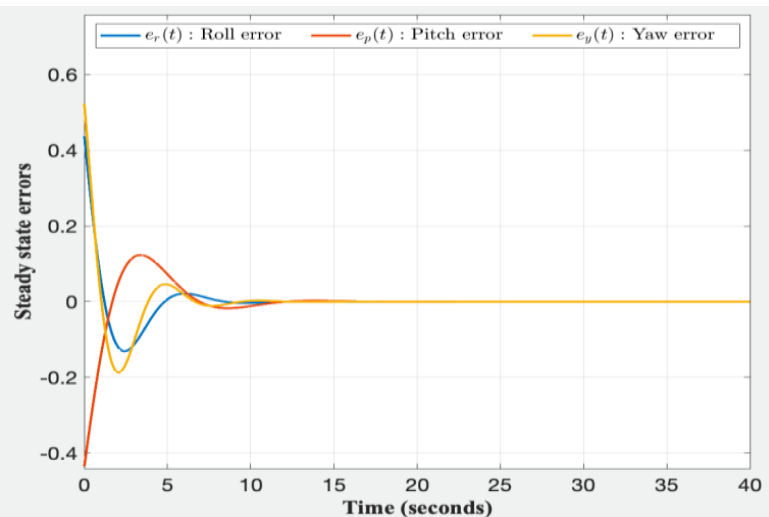

Figure. 15. Response of stead error of Roll, Pitch, Yaw

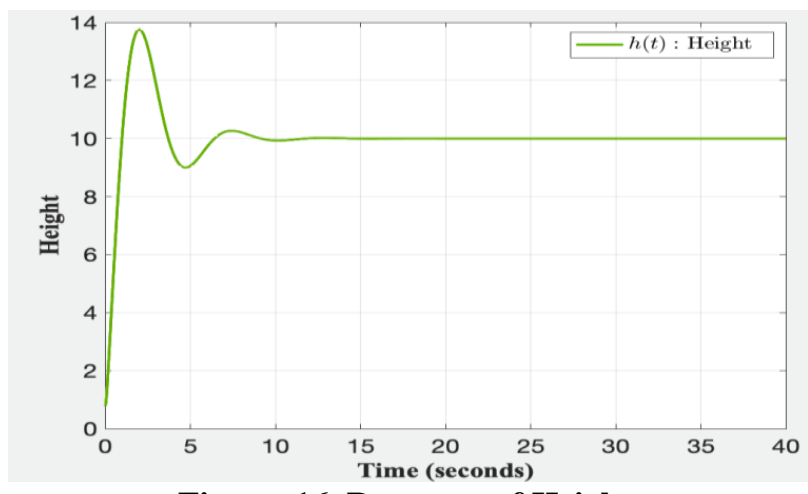

Figure. 16. Response of Height.

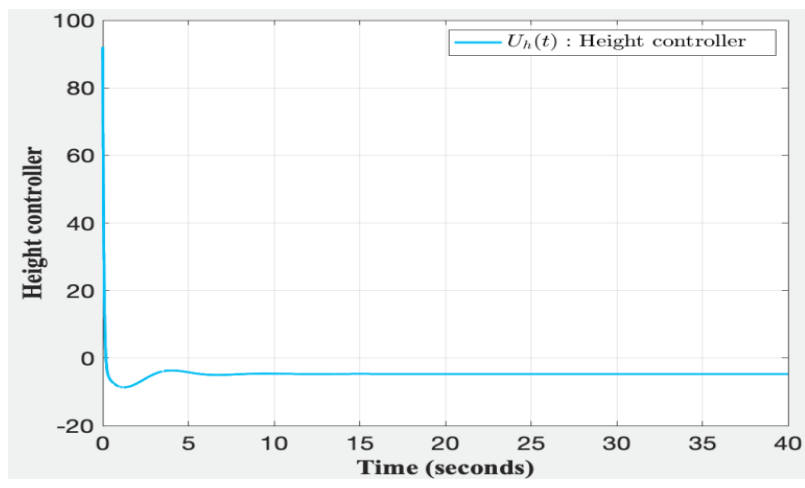

Figure. 17. Response of Height controller
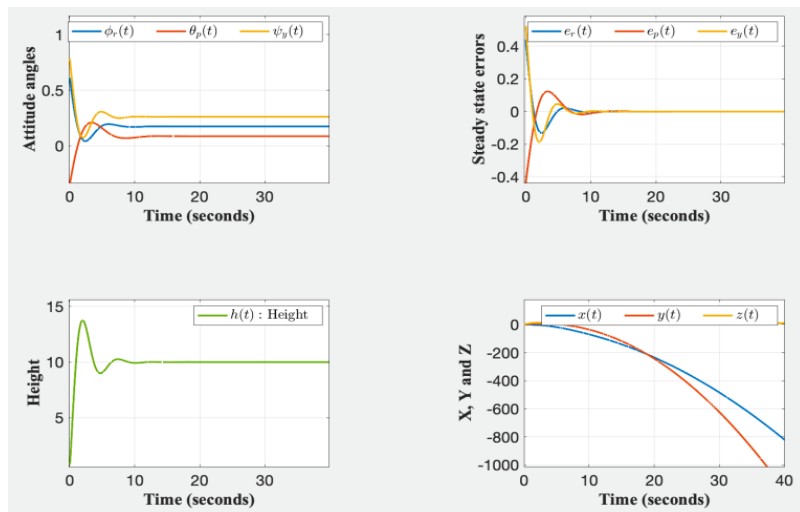

Figure. 18. Results of Stead state error

C. Response of the Decoupled Multivariable Qua-drone system

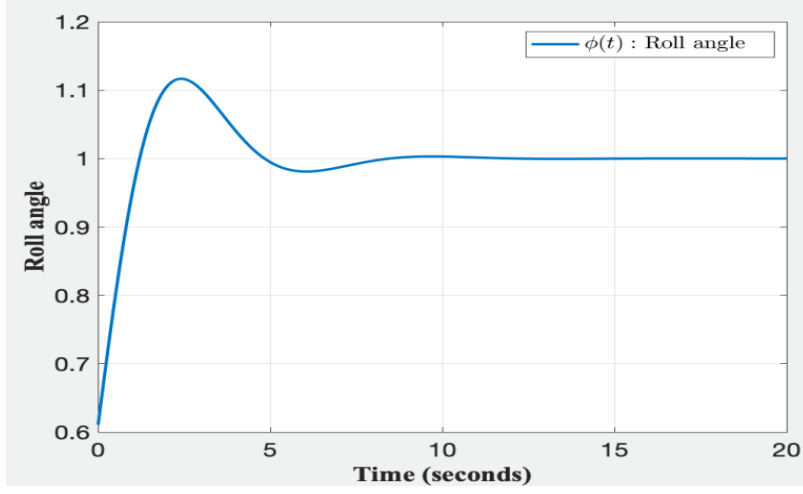

Figure. 19. Roll angle in decoupled qua-drone system

Published By:

Blue Eyes Intelligence Engineering

and Sciences Publication (BEIESP)

DOI: 10.35940/ijrte.D6627.1110421

(C) Copyright: All rights reserved.

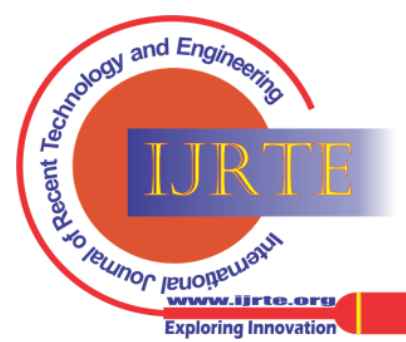




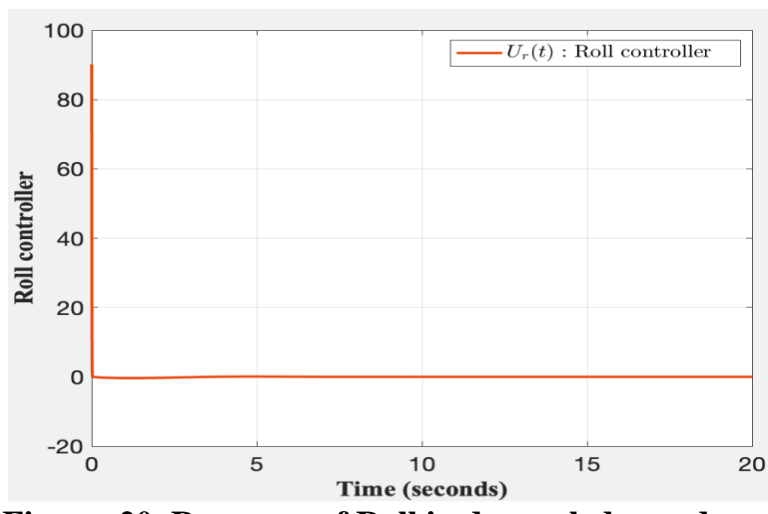

Figure. 20. Response of Roll in decoupled qua-drone system

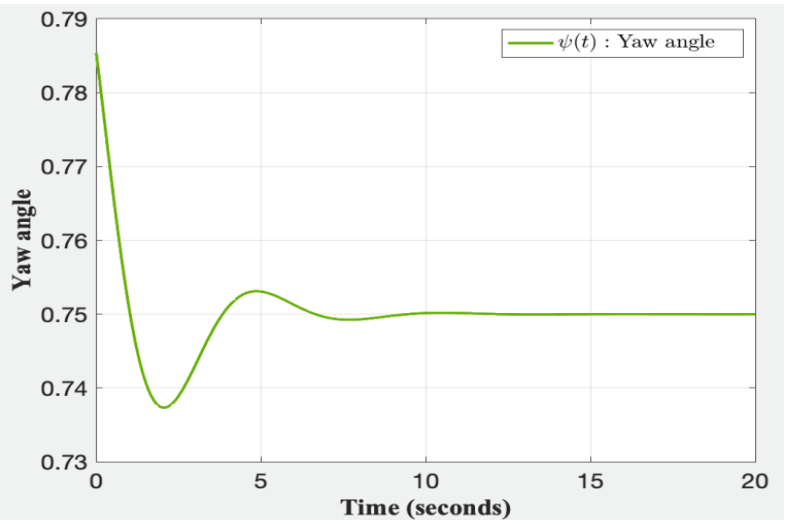

Figure. 21. Response of Yaw angle decoupled qua-drone system

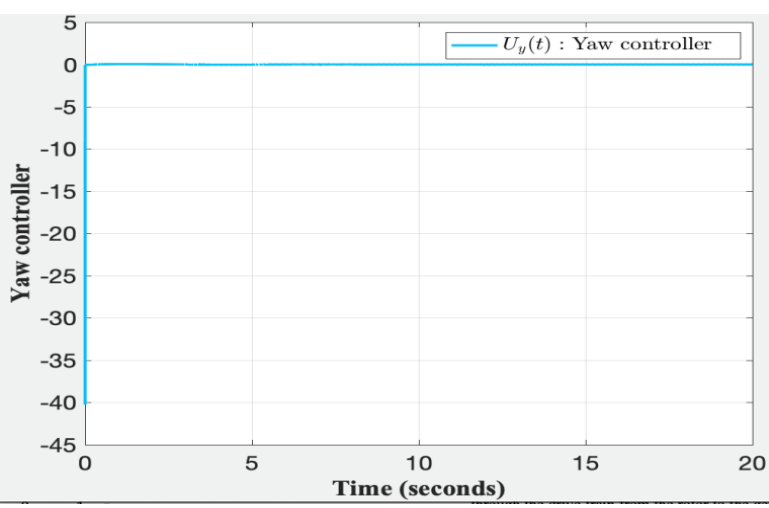

Figure. 22. Response of Yaw controller in decoupled qua-drone system

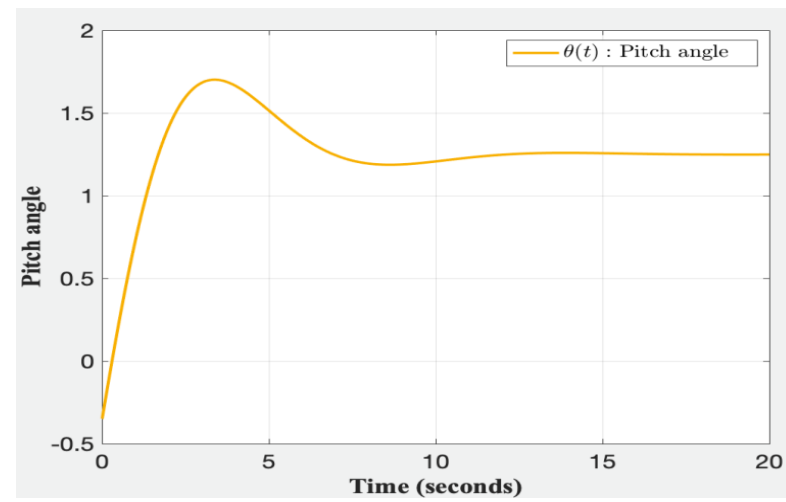

Figure. 23. Response of Pitch angle in decoupled quadrone system

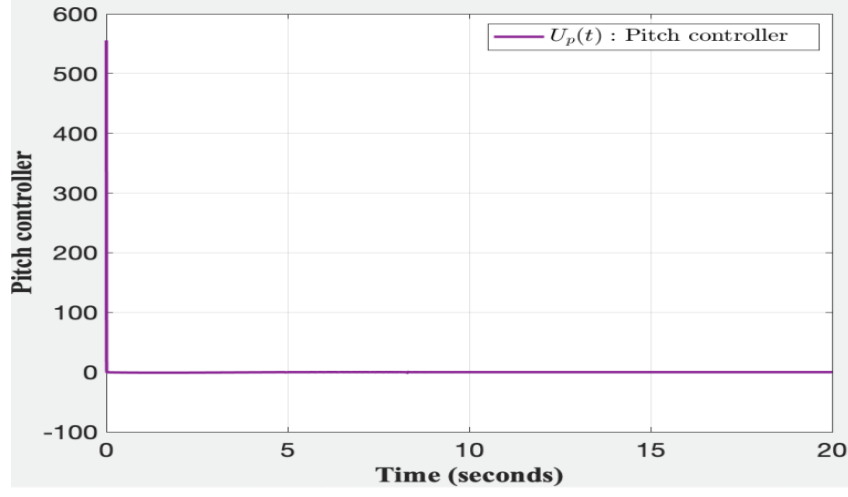

Figure. 24. Response of Pitch controller in decoupled qua-drone system

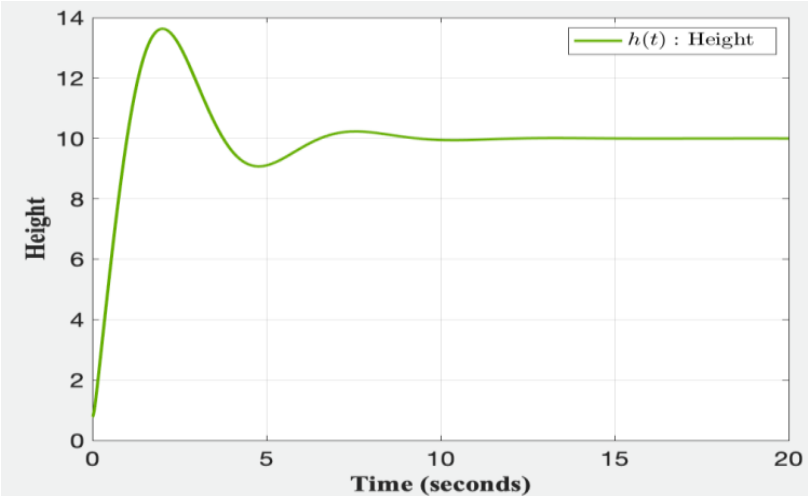

Figure. 25. Response of Height in decoupled qua-drone system

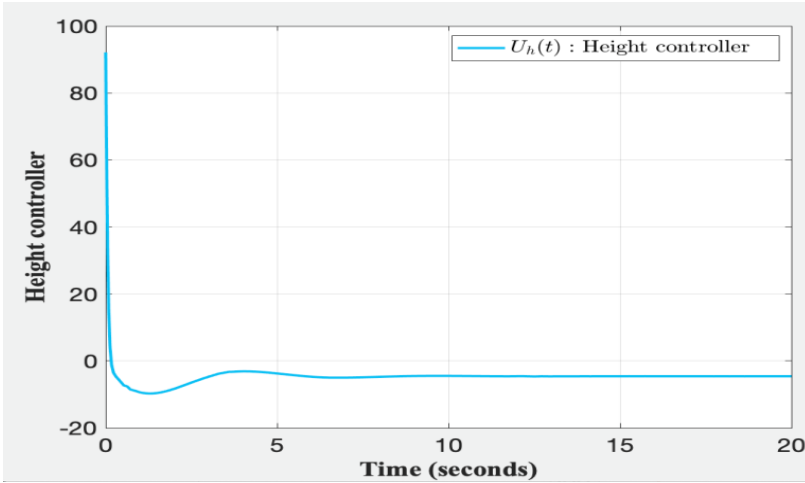

Figure. 26. Response of Height controller in decoupled qua-drone system

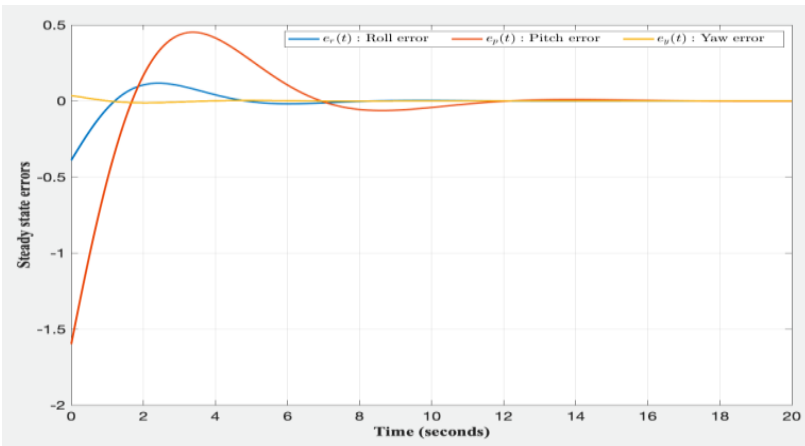

Figure. 27. Response of stead state error in decoupled qua-drone system

Published By:

Blue Eyes Intelligence Engineering and Sciences Publication (BEIESP) 

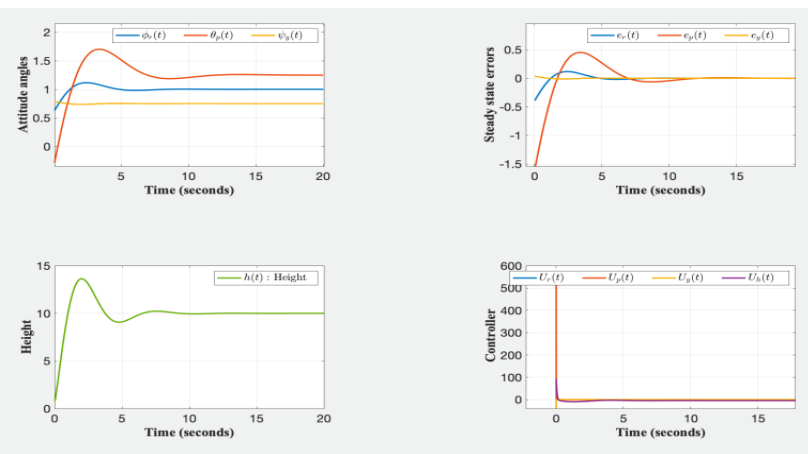

Figure. 28. Response of stead state in decoupled quadrone system

Figure 19-28 illustrates the response of the decoupled multivariable qua-drone. From Figure 9-18 of the uncoupled multivariable qua-drone and Figure 19-28 of the decoupled multivariable qua-drone, we can see the response of dynamic response is different in some responses. For instance, Figure 15 and 27 have quite different response and Figure 18 and Figure 28 have also much different results. However, others are almost the same response. It means that this multivariable qua-drone has the similar response in some characteristics but illustrates a different expression in some parts because of the coupled characteristics. Because of this coupled characteristic of some parts, we have to have a decoupling process for multivariable qua-drones.

\section{Intelligent Optimal Tuning of PID Controller of the Decoupled Multivariable Qua-drone System}

GA based Tuning

In this section, this paper tunes using GA (Genetic Algorithm) for the multivariable qua-drone decoupled system.

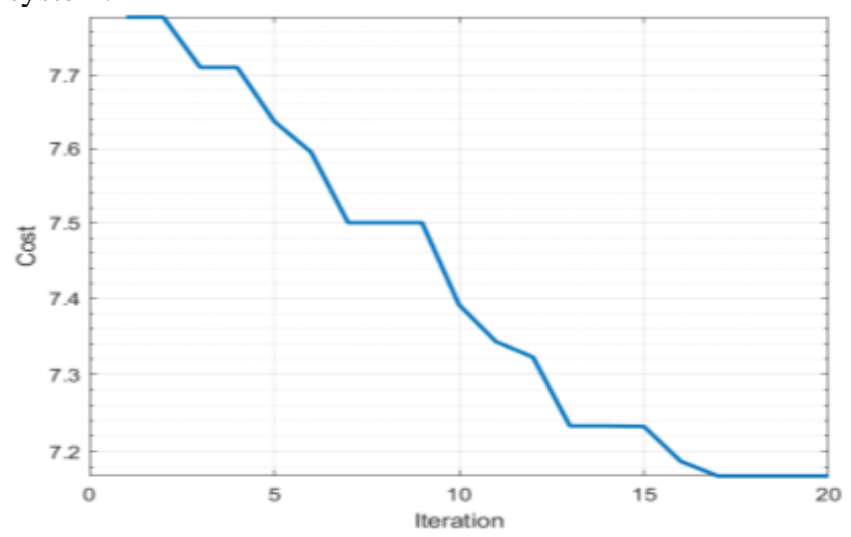

Figure. 29. Response of GA optimization (Iteration Vs.

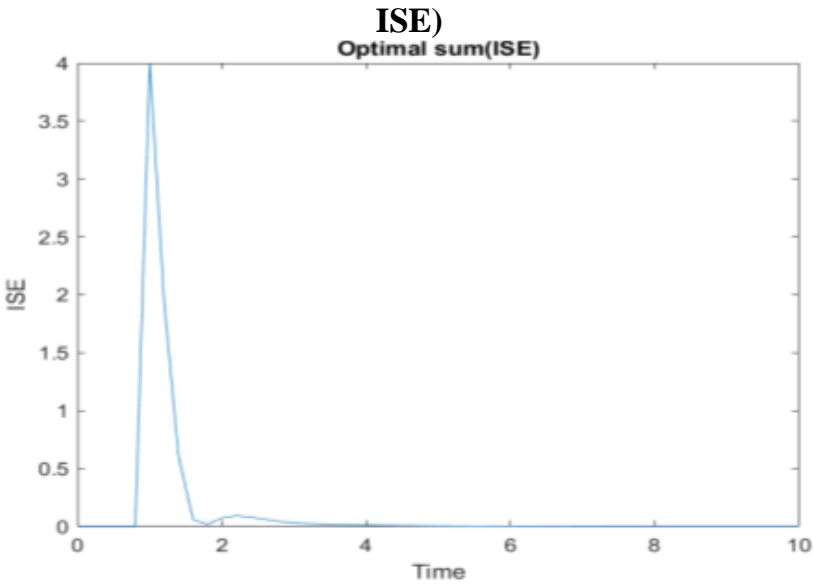

Figure. 30. Response of GA sum of all error

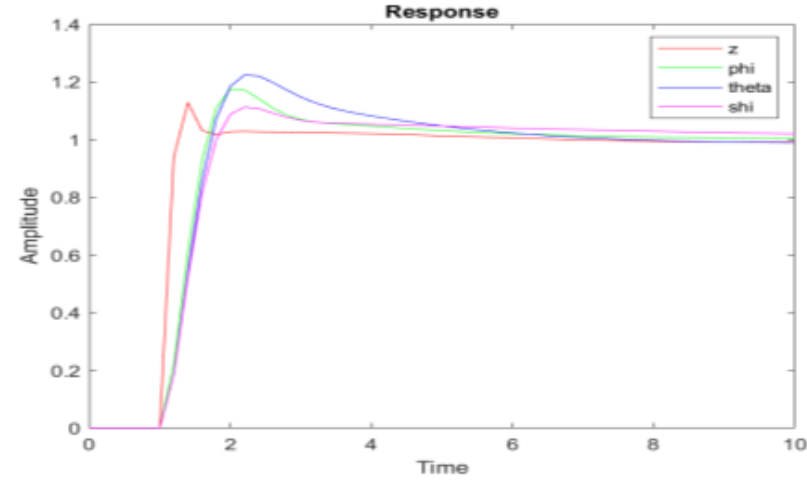

Figure. 31. Step response by GA optimization (Step Response for Z-axis, phi: Roll angle, theta: Pitch angle, shi: Yaw angle)

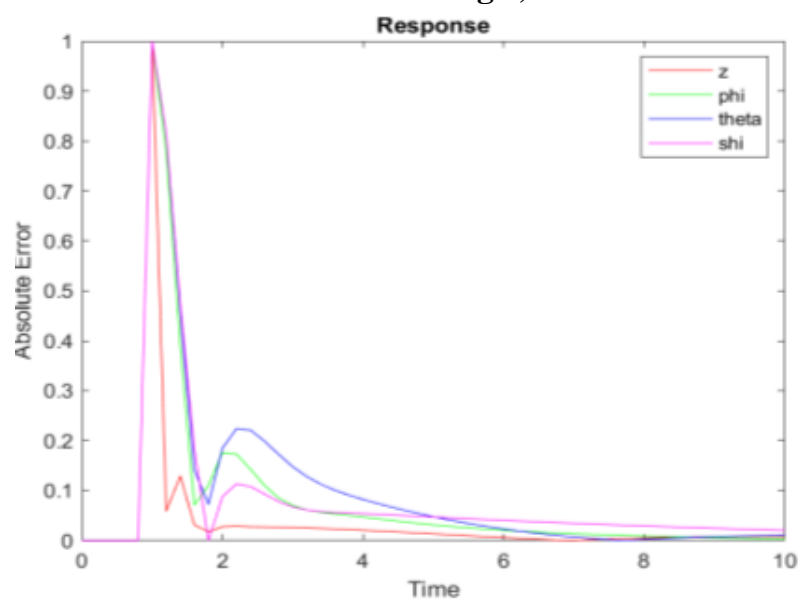

Figure. 32. Response of GA optimization (Step Response for Z-axis, phi: Roll angle, theta: Pitch angle, shi: Yaw angle)

Table- I: Tuning Specification Obtained by GA.

\begin{tabular}{|c|c|c|c|c|}
\hline Item & Z-axis & $\begin{array}{c}\text { Phi } \\
\text { (Roll angle) }\end{array}$ & $\begin{array}{c}\text { Theta } \\
\text { (Pitch angle) }\end{array}$ & $\begin{array}{c}\text { Shi } \\
\text { (Yaw angle) }\end{array}$ \\
\hline Rise Time & 0.1686 & 0.4930 & 0.5246 & 0.6012 \\
\hline SettlingTime & 5.3098 & 5.6420 & 6.8273 & 5.9406 \\
\hline SettlingMin & 0.9410 & 0.9289 & 0.9897 & 1.0002 \\
\hline SettlingMax & 1.1291 & 1.1758 & 1.2241 & 1.1134 \\
\hline Overshoot & 13.9002 & 17.1214 & 23.6824 & 9.1022 \\
\hline Undershoot & 0 & 0 & 0 & \\
\hline Peak & 1.1291 & 1.1758 & 1.2241 & 1.1134 \\
\hline PeakTime & 1.4000 & 2 & 2.2000 & 2.2000 \\
\hline
\end{tabular}

Figure 29-32 shows the results of GA-based tuning. Figure 29 is response of GA optimization (Iteration Vs ISE) and Figure 30 presents response of GA sum of all error. Figure 31 shows step response by GA optimization. Depending on parameters, we can obtain several responses and it is quite convenient to tune because whenever we want different tuning response, we can adjust using simple changing of one value. Table- $\mathrm{I}$ is the tuning specification obtained by using GA.

Published By:

Blue Eyes Intelligence Engineering and Sciences Publication (BEIESP) 
PSO Based Tuning

Figure 33-36 shows the results obtained by PSO. Figure 33 illustrates response to PSO optimization (Iteration Vs. ISE) and Figure 34 illustrates response of PSO sum of all error.

Figure 35 shows step response by PSO optimization. Response of PSO optimization (Step Response for Z, phi, theta, shi)

Figure 36 is the response of PSO optimization (Step Response for Z-axis, phi (Roll angle), theta (Pitch angle), shi (Yaw angle)

Table-II shows tuning specifications obtained by using PSO.

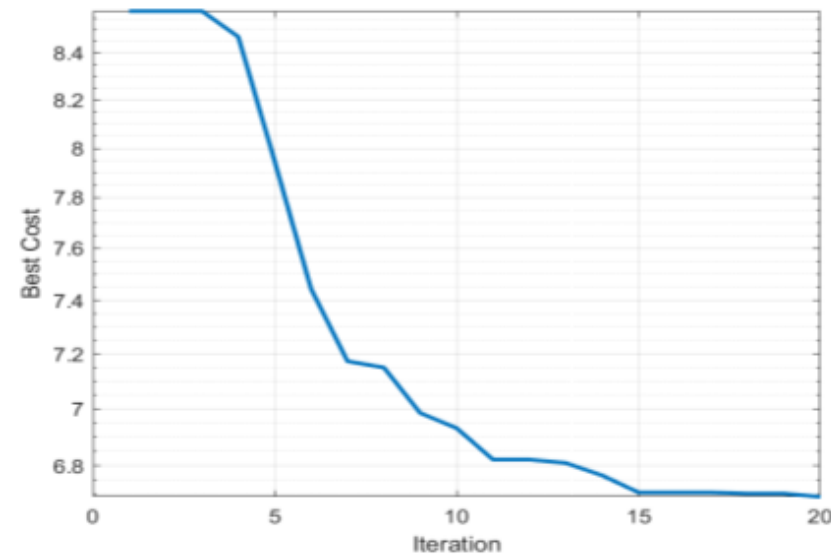

Figure. 33. Response of PSO optimization (Iteration Vs. ISE)

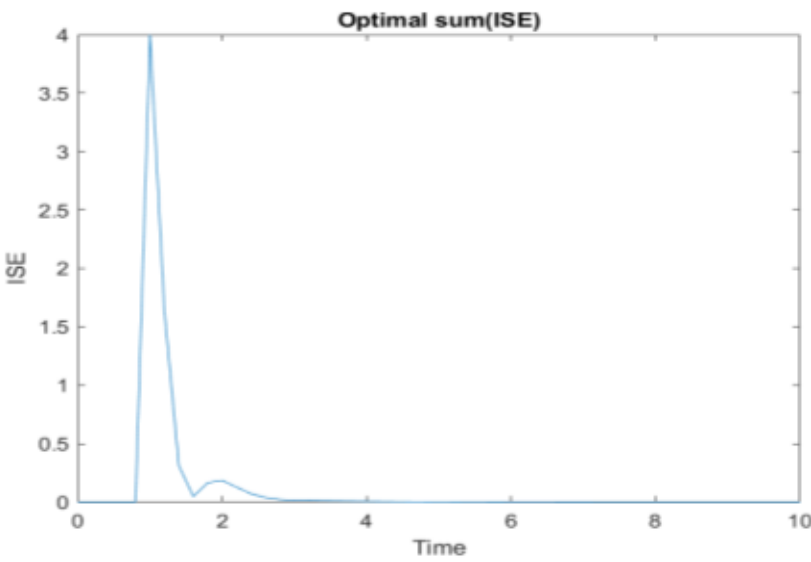

Figure. 34. Response of PSO sum of all error

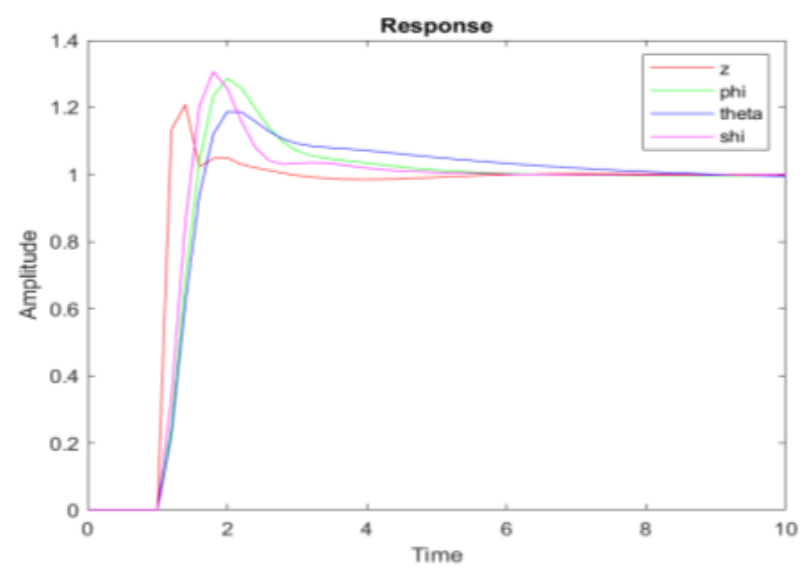

Figure. 35. Step response by PSO optimization (Z-axis, phi: Roll angle, theta: Pitch angle, shi: Yaw angle)

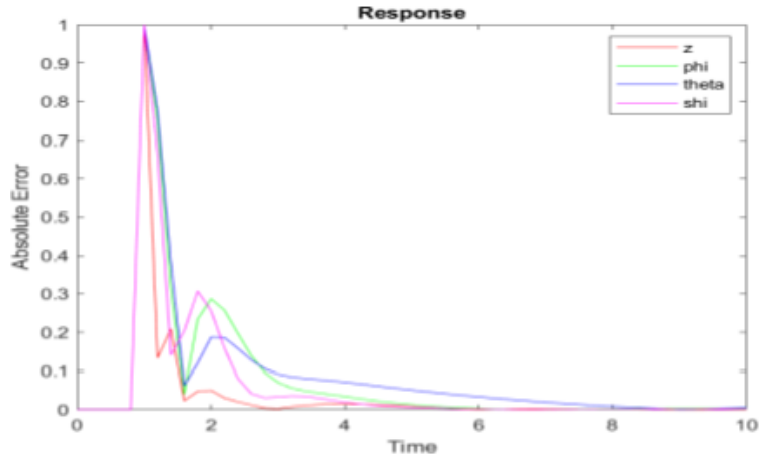

Figure. 36. Response of PSO optimization (Z-axis, phi: Roll angle, theta: Pitch angle, shi: Yaw angle)

Table- II: Tuning Specification Obtained by PSO.

\begin{tabular}{|c|c|c|c|c|}
\hline Item & Z-axis & $\begin{array}{c}\text { Phi } \\
\text { (Roll angle) }\end{array}$ & $\begin{array}{c}\text { Theta } \\
\text { (Pitch angle) }\end{array}$ & $\begin{array}{c}\text { Shi } \\
\text { (Yaw angle) }\end{array}$ \\
\hline Rise Time & 0.141 & 0.4389 & 0.4847 & 0.3636 \\
\hline Settling Time & 2.4212 & 4.7666 & 7.3720 & 4.0264 \\
\hline Settling Min & 0.9852 & 0.9968 & 0.9372 & 0.9985 \\
\hline Settling Max & 1.2081 & 1.2869 & 1.1872 & 1.3071 \\
\hline Overshoot & 20.8564 & 29.1015 & 19.3138 & 30.9002 \\
\hline Undershoot & & 0 & 0 & 0 \\
\hline Peak & 1.2081 & 1.2869 & 1.1872 & 1.3071 \\
\hline Peak Time & 1.4000 & 2 & 2 & 1.8000 \\
\hline
\end{tabular}

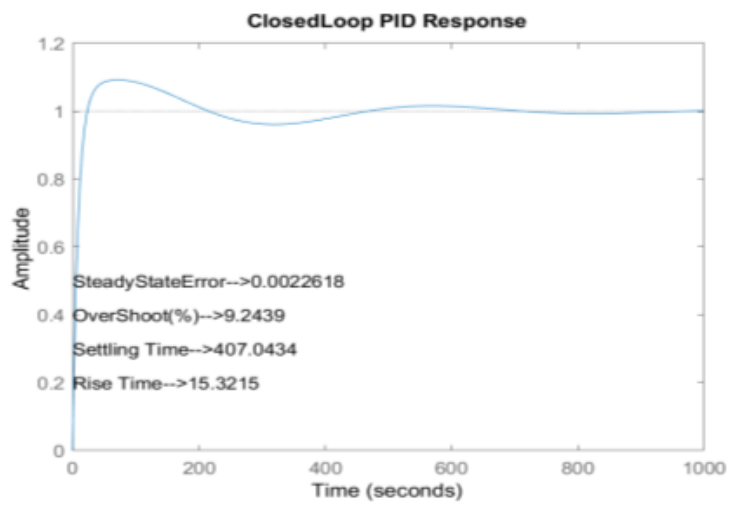

Figure. 37. Response of closed loop by PSO optimization

Table- III: Tuning Specification Obtained by ZieglerNichols.

\begin{tabular}{|c|c|c|c|c|}
\hline Item & Z-axis & $\begin{array}{c}\text { Phi } \\
\text { (Roll } \\
\text { angle) }\end{array}$ & $\begin{array}{c}\text { Theta } \\
\text { (Pitch } \\
\text { angle) }\end{array}$ & $\begin{array}{c}\text { Shi } \\
\text { (Yaw angle) }\end{array}$ \\
\hline Rise Time & 15.0027 & 15.0027 & 15.0027 & 15.0027 \\
\hline Settling Time & 413.113 & 413.113 & 413.113 & 413.113 \\
\hline Settling Min & 0.9190 & 0.9190 & 0.9190 & 0.9190 \\
\hline Settling Max & 1.0923 & 1.0923 & 1.0923 & 1.0923 \\
\hline Overshoot & 9.0861 & 9.0861 & 9.0861 & 9.0861 \\
\hline Undershoot & 0 & 0 & 0 & 0 \\
\hline Peak & 1.0923 & 1.0923 & 1.0923 & 1.0923 \\
\hline Peak Time & 74.9170 & 74.9170 & 74.9170 & 74.9170 \\
\hline
\end{tabular}

Published By:

Blue Eyes Intelligence Engineering and Sciences Publication (BEIESP) 


\section{Dynamic Decoupling and Intelligent Optimal PID Controller Tuning of Multivariable Qua-drones}

\section{V.CONCLUSION}

This paper deals with intelligent optimal PID controller tuning of multivariable qua-drone by using Ziegler-Nichols, GA (Genetic Algorithm, and PSO (Particle Swarm Optimization). Basically, aviation systems and robot systems including drones have a highly nonlinear dynamic characteristic and complex multivariable systems. Therefore, the tuning of these control systems is not easy because of interacting behavior among parameters. There are many approaches to overcome these complex problems, but many cases are single loop approaches. In the case of multivariable, they study chemical or plant. Even though several intelligent theories for tuning have been developed significantly, there are still some problems in application in case of high speed such as motor drives and flight control. Many controllers are poorly tuned to these practices. To get over this problem, this paper focuses on the intelligent problem of multivariable qua-drones. To do that, firstly, this paper decouples multivariable characteristics of qua-drone to a single loop and then performs intelligent tuning by GA and PSO to obtain optimal response and compare these results to find which tuning method is better.

\section{ACKNOWLEDGEMENT}

This work was supported by the National Research Foundation of Korea (NRF) grant funded by the Korea government (MSIT) (No. 2021056145). Author thanks to support of Kore government (MSIT).

\section{REFERENCES}

1. Liu, G. P., \& Daley, S., "Optimal-tuning PID controller design in the frequency-domain with application to rotary hydraulic systems," Control Engineering Practice, 7, pp. 821-830, 1999.

2. McCormack, A. S., \& Godfrey, K., "Rule-based auto-tuning based on frequency domain identification," IEEE Transactions on Control Systems Technology, 6(1), 43-61, 1998.

3. Bernardo Sousa Machado Henriques, "Estimation and Control of a Quadrotor Attitude", 2011.

4. Sevkuthan KURAK1, "Control and Estimation of a Quadcopter Dynamical Model", Periodicals of Engineering and Natural Sciences ISSN 2303-4521 Vol.6, No.1, March 2018, pp. 63-75, Available online at: http://pen.ius.edu.ba.

5. Oguz Kose et el. "Dynamic Modeling and Simulation of Quadrotor for Different Flight Conditions", European Journal of Science and Technology No. 15, pp. 132-142, March 2019.

6. C. C. Hang and K. J. Astrom, "Refinements of the Ziegler-Nichols tuning formular, "Proc. Inst. Elect. Eng., Vol. 138, pt. D, pp. 111118, 1991.

7. Dong Hwa Kim, "Experimental Research of Intelligent Multivariable 2-DOF PID Control System for DCS," International Journal of Systems Applications, Engineering \& Development, July 2013, pp. 148-157.

8. D. H. Kim, "Application of a multivariable PID controller with a neural network tuning method to the heat exchange," FUZZ-IEEE, pp. 23-25, Aug.1998, Seoul.

9. Dong Hwa Kim, "Multiobjective PI controller tuning of multivariable boiler control system using immune algorithm," KFIS, Vol. 3, No. 1, pp.78-86, 2003.

10. Dong Hwa Kim, "A Study on Improving Lecture Skill and Implementation of Anti-reset and Bampless Using 2-DOF-PID Controller and Python IARJSET, Vol. 7, Issue 10, October 2020, 2394-1588.

11. C. H. Lee and C. C. Teng, "A Novel Robust PID Controllers Design by Fuzzy Neural Network," Asian Journal of Control, Vol. 4, No. 4, pp. 433-438, 2002.

12. Jemie Muliadi, "Neural Network Control System of UAV Altitude Dynamics and Its Comparison with the PID Control System," Journal of Advanced Transportation Volume 2018.

13. Moussa Labbadi, et el, "Robust Integral Terminal Sliding Mode
Control for Quadrotor UAV with External Disturbances,' International Journal of Aerospace Engineering, Volume 2019, $\begin{array}{llll}\text { Article ID } & 2016416, & 1-10 & \text { pages, }\end{array}$ https://doi.org/10.1155/2019/2016416

14. Dong Hwa Kim, "Decentralized PID Controller Tuning for Multivariable Process Using Multiobjective Optimization Based on Bacterial Foraging," IEEE 2005 Intelligent computing, Lecture Notes in Computer Science Proceeding of Springer (SCI), Aug. 2326, 2005, China.

15. Ramiro Ibarra Pérez, et el, "Attitude Control of a Quadcopter Using Adaptive Control Technique,"

16. https://www.intechopen.com/chapters/57334.

17. Wojciech Giernacki, et el, "Mathematical Modeling of the Coaxial Quadrotor Dynamics for Its Attitude and Altitude Control," J. Mathematical Modeling of the Coaxial Quadrotor Dynamics for Its Attitude and Altitude Control. Energies 2021, 14, 1232. https:// doi.org/10.3390/en14051232.

18. https://www.techlearning.com/buying-guides/best-droneforeducation-2020.

19. Batıkan E Demir, et el, "Real-time trajectory tracking of an unmanned aerial vehicle using a self-tuning fuzzy proportional integral derivative controller," International Journal of Micro Air Vehicles, Vol. 8(4) 252-268, 2016.

20. J. G. Ziegler and N. B. Nichols, "Optimum Settings for Automatic Controllers,” Trans. ASME, Vol. 64, pp. 759-768, 1942.

21. C. H. Lee and C. C. Teng, "Tuning of PID Controllers for Stable and Unstable Processes Based on Gain and Phase Margin Specifications: A Fuzzy Neural Approach,” International Journal of Fuzzy Systems, Vol. 3, No. 1, pp. 346-355, 2001.

22. Sevkuthan KURAK et el, "Control and Estimation of a Quadcopter Dynamical Model," Periodicals of Engineering and Natural Sciences, Vol.6, No.1, March 2018, pp. 63-75.

23. Oguz Kose, et. El, "Dynamic Modeling and Simulation of Quadrotor for Different Flight Conditions," European Journal of Science and Technology No. 15, pp. 132-142, March 2019.

24. Anežka Chovancová, et.el, "Mathematical Modelling and Parameter Identification of Quadrotor, ScienceDirect (Procedia Engineering), 96 (2014) $172-181$.

25. S. Jamebozorg, "Multivariable Decoupling Control System Based on Generalized Diagonal Dominance," International Journal of Electronics Communication and Computer Engineering, Volume 6, Issue 2, 2015

26. Rui Ma, et el, "Dynamic Decoupling Control Optimization for a Small-Scale Unmanned Helicopter," Journal of Robotics, Volume 2018, Article ID 9897684, 12 pages.

27. Anuradha Purty, et. El, "Comparative Study of Decoupler with Different Controllers for Two Inputs and Two Output System," International Journal of Innovative Research in Science, Engineering and Technology, Vol. 6, Issue 1, January 2017.

28. N. ROY, S. MENSAH, "Design of a multivariable controller for a CANDU 600MW Nuclear power plant using the INA method, AECL-8342, 1984

29. Hamid Saeed Khan, et el, "Attitude and Altitude Control of Quadrotor by Discrete PID control and Non-linear Model Predictive Control,” https://www.researchgate.net/publication/304701608, 2015.

30. J. López, et el, “A Robust $\mathrm{H} \infty$ Controller for an UAV Flight Control System, "Scientific World Journal. 2015; 2015.

31. Dong Hwa Kim, "Robust tuning of PID controllers with disturbance rejection using bacterial foraging-based optimization," WSEAS Transaction on systems Vol. 3, No. 9, 2004, pp. 2834-2840.

32. Dong Hwa Kim, Jae Hoon Cho, "Robust PID using Gain/Phase margin and advanced immune algorithm," WSEAS Transaction on systems Vol. 3, No. 9, 2004, pp. 2841-2851.

33. Gabriel M. Hoffmann," Quadrotor Helicopter Flight Dynamics and Control: Theory and Experiment," AIAA Guidance, Navigation and Control Conference and Exhibit, 20 - 23 August 2007, pp. 1-20, 2007.

34. Xiaodong Zhang, "A Survey of Modelling and Identification of Quadrotor Robot," Volume 2014, Article ID 320526, pp. 1-16, 2014.

35. Dewei Zhang," The Quadrotor Dynamic Modeling and Indoor Target Tracking Control Method," Volume 2014, Article ID 637034, 9 pages http://dx.doi.org/10.1155/2014/637034, 2014. 
36. Aurelio Bermu dez et el, "Drone challenge: A platform for promoting programming and robotics skills in K-12 education," International J. of advanced robot systems, 2018, http://www.creativecommons.org/licenses/by/4.0/)

37. Rahul Abasaheb Bhosale, "Mathematical Modeling and Simulation of Quadcopter-UAV Using PID Controller," IOSR Journal of Engineering (IOSRJEN), PP 29-36, 2018.

38. Nguyen Xuan-Mung, "Improved Altitude Control Algorithm for Quadcopter Unmanned Aerial Vehicles,” Appl. Sci. Vol. 9, 2019.

39. Ayad Al-Mahturi, "Nonlinear Altitude Control of a Quadcopter Drone Using Interval Type-2 Fuzzy Logic,” IEEE Symposium Series on Computational Intelligence SSCI, pp. 236-241, 2018.

40. Jemie Muliadi and Benyamin Kusumoputro, "Neural Network Control System of UAV Altitude Dynamics and Its Comparison with the PID Control System," Journal of Advanced Transportation Volume 2018, Article ID 3823201, pp. 1-18, 2018.

41. Min Xue, "UAV Trajectory Modeling Using Neural Networks," https://ntrs.nasa.gov/search.jsp?R=20170009832 2020-0104T12:53:39+00:00Z.

42. A. Van Wyk, "Quadcopter Control using Intelligent Control Methods," https://www.researchgate.net/publication/325999949, 2017.

43. OsmanÇakir, "Neural Network Control for Quadrotors," American Scientific Research Journal for Engineering, Technology, and Sciences (ASRJETS), pp.191-200, http://asrjetsjournal.org/

44. Jemin Hwangbo, "Control of a Quadrotor with Reinforcement Learning," IEEE ROBOTICS AND AUTOMATION LETTERS. PREPRINT VERSION, pp. 1-8, June, 2017.

45. DivyaJoshi, "Drone technology uses and applications for commercial, industrial and military drones in 2020 and the future,"

46. R. Mahony, V. Kumar, and P. Corke, "Multirotor aerial vehicles modeling, estimation, and control of quadrotor," IEEE Robotics and Automation Magazine, vol. 19, no. 3, pp. 20-32, 2012.

47. P. Pounds, R. Mahony, and P. Corke, "Modelling and control of a large quadrotor robot," Control Engineering Practice, vol. 18, no. 7, pp. 691-699, 2010.

48. J. H. Kim, M.-S. Kang, and S. Park, "Accurate modeling and robust hovering control for a quad-rotor VTOL aircraft," Journal of Intelligent and Robotic Systems, vol. 57, no. 1-4, pp. 9-26, 2010.

49. H. Lim, J. Park, D. Lee, and H. J. Kim, "Build your own quadrotor: Opensource projects on unmanned aerial vehicles," IEEE Robotics and Automation Magazine, vol. 19, no. 3, pp. 33- 45, 2012.

50. D. Mellinger and V. Kumar, "Minimum snap trajectory generation and control for quadrotors," in Proceedings of the IEEE International Conference on Robotics and Automation (ICRA '11), pp. 2520-2525, IEEE, Shanghai, China, May 2011.

51. Q. Zhan, J. Q. Wang, and X. Xi, "Control system design and experiments of a quadrotor," in Proceedings of the IEEE International Conference on Robotics and Biomimetics (ROBIO '12) pp. 1152-1157, IEEE, Guangzhou, China, December 2012.

52. L. Jun and Y. T. Li, "Dynamic analysis and PID control for a quadrotor," in Proceedings of the International Conference on Mechatronics and Automation, pp. 573-578, IEEE, Beijing, China, August 2011.

53. A. L. Salih, M. Moghavvemi, H. A. F. Mohamed, and K. S. Gaeid, "Flight PID controller design for a UAV quadrotor," Scientific Research and Essays, vol. 5, no. 23, pp. 3660-3667, 2010.

54. A. B. Milhim, Y. Zhang, and C. A. Rabbath, "Quadrotor UAV-high fidelity modeling and nonlinear PID control," in Proceedings of AIAA Modeling and Simulation Technologies Conference, pp. 2010-8362, Toronto, Canada, 2010.

55. M. Santos, V. Lo' pez, and F. Morata, "Intelligent fuzzy controller of a quadrotor," in Proceedings of the IEEE International Conference on Intelligent Systems and Knowledge Engineering (ISKE '10), pp. 141-146, IEEE, Hangzhou, China, November 2010.

56. C. Vlachos, "Genetic approach to decentralized PI controller tuning for multivariable processes," IEE Proc.-Control Theory Appl., Vol. 146, No. 1, pp. 58-64, March 1999.

57. Vrancic, "Tuning of decoupling controller by using MOMI method," Report, Dept. of Computer Automation and Control, Jozef Stefan Institute, Solvenia.

58. Dong Hwa Kim, "Robust Intelligent Tuning of PID Controller for Multivarlable System Using Clonal Selection and Fuzzy Logic," KES2005, Melbourne, 2005.

59. Wang, Q.G., B. Huang, X. Guo, “Auto-tuning of TITO decoupling controllers from step tests", ISA Transactions, Vol. 39, pp. 407-418, 2000.

60. Reato A. Krohling and joost P.Rey, "Design of Optimal Disturbance Rejection PID Controllers Using Genetic Algorithms," IEEE Trans. Evolutionary and computation. Vol. 5, No. 1, Feb. 2001.
61. Dong Hwa Kim and Jae Hoon Cho, "Intelligent tuning of PID Controller with disturbance rejection function using immune algorithm," IEEE-CIMSA2004 conference, 2004.

62. Heba talla Mohamed Nabil ElKholy, "Dynamic Modeling and Control of a Quadrotor Using Linear and Nonlinear Approaches," The American University in Cairo (AUC), Master thesis, 2014.

63. ELMER G. GILBERT, "The decoupling of multivariable system by state feedback," SIAM J. CONTROL, Vol. 7, No. 1, February 1969.

64. E. Okyere, "LQR controller design for Qua-rotor helicopters," The Journal of Engineering, June 2018.

65. N. Koksal et el, M, "Adaptive Linear Quadratic Attitude Tracking Control of a Quadrotor UAV Based on IMU Sensor Data Fusion, October 2018.

66. Sevkuthan KURAK et el, "Control and Estimation of a Quadcopter Dynamical Model," Periodicals of Engineering and Natural Science Vol.6, No.1, pp. 63-75, 2018

67. N. Koksal, et el, "Adaptive Linear Quadratic Attitude Tracking Control of a Quadrotor UAV Based on IMU Sensor Data Fusion," Sensors Vol. 19, No. 46, 2019.

68. Martín A, et el, "Trajectory planning for an unmanned quadrotor," https://www.researchgate.net/publication/315734938, Conference, Nov. 2016.

69. Zeng-Rong Hu, "Analytical Design of PID Decoupling Control for TITO Processes with Time Delays," Journal of computers, Vol. 6, No. 6, June 2011.

70. Ziyang Zhen, "Information Fusion Based Decoupling Control for Multivariable Nonlinear System," Mathematical Problems in Engineering Vol. 2015, Article ID 361581, 10 pages http://dx.doi.org/10.1155/2015/361581

\section{AUTHORS PROFILE}

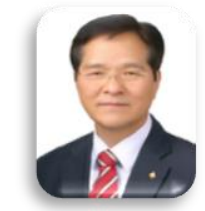

Dong Hwa Kim Science, Interdisciplinary Graduate School of Science and Engineering (AI Application for Automatic control), TIT (Tokyo Institute of Technology), Tokyo, Japan. He worked the Hanbat National University as Prof. (Dean, S. Korea), He has experience in many Universities, overseas as Prof. Electrical Power and Control Eng. Adama Science and Tech. Uni. Ethiopia. Vietnam TDT (Director, establish Korea Expert Center), Mongolian University (Dean, Graduate school). He ever worked as NCP of EU-FP7 (EU-Framework Program, ICT). He had keynote speak at several international conferences and Universities. He has 200 papers in Journal and conferences. He worked editor of IJCIR (International Journal of Computational Intelligence) and He is reviewing IEEE and other's Journal.

Home page: www.worldhumancare.wixsite.com/kimsite

Research citations: https://www.researchgate.net/profile/Dong Kim53

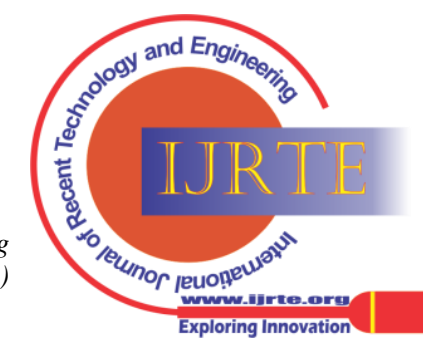

